\title{
AN ALMA SURVEY OF SUBMILLIMETER GALAXIES IN THE EXTENDED CHANDRA DEEP FIELD SOUTH: NEAR-INFRARED MORPHOLOGIES AND STELLAR SIZES
}

\author{
Chian-Chou Chen (陳建州) $)^{1}$, Ian Smail ${ }^{1}$, A. M. Swinbank ${ }^{1}$, J. M. Simpson ${ }^{1}$, Cheng-Jiun Ma $^{1}$, D. M. Alexander ${ }^{1}$,

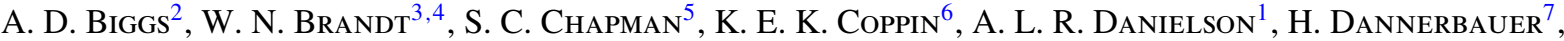

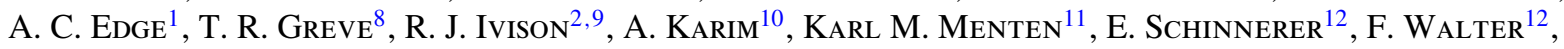 \\ J. L. WARDLOW ${ }^{13}$, A. WEIß ${ }^{11}$, AND P. P. VAN DER WERF ${ }^{14}$ \\ ${ }^{1}$ Institute for Computational Cosmology, Durham University, South Road, Durham DH1 3LE, UK \\ ${ }_{2}^{2}$ European Southern Observatory, Karl Schwarzschild Strasse 2, D-85748 Garching, Germany \\ ${ }^{3}$ Department of Astronomy and Astrophysics, 525 Davey Lab, Pennsylvania State University, University Park, PA 16802, USA \\ ${ }^{4}$ Institute for Gravitation and the Cosmos, Pennsylvania State University, University Park, PA 16802, USA \\ ${ }^{5}$ Department of Physics and Atmospheric Science, Dalhousie University, Halifax, NS B3H 3J5, Canada \\ ${ }^{6}$ Centre for Astrophysics Research, Science and Technology Research Institute, University of Hertfordshire, Hatfield AL10 9AB, UK \\ ${ }^{7}$ Institut für Astrophysik, Universität Wien, Türkenschanzstraße 17, A-1180 Wien, Austria \\ ${ }^{8}$ Department of Physics and Astronomy, University College London, Gower Street, London WC1E 6BT, UK \\ ${ }^{9}$ Institute for Astronomy, University of Edinburgh, Royal Observatory, Blackford Hill, Edinburgh EH9 3HJ, UK \\ ${ }^{10}$ Argelander-Institute for Astronomy, Bonn University, Auf dem Hügel 71, D-53121 Bonn, Germany \\ ${ }^{11}$ Max-Planck-Institut für Radioastronomie, Auf dem Hügel 69, D-53121 Bonn, Germany \\ ${ }_{12}$ Max-Planck Institute for Astronomy, Königstuhl 17, D-69117 Heidelberg, Germany \\ ${ }^{13}$ Dark Cosmology Centre, Niels Bohr Institute, University of Copenhagen, DK-2100 Copenhagen, Denmark \\ ${ }^{14}$ Leiden Observatory, Leiden University, P.O. Box 9513, 2300 RA Leiden, The Netherlands \\ Received 2014 August 19; accepted 2014 November 25; published 2015 January 29
}

\begin{abstract}
We analyze Hubble Space Telescope WFC3/H160-band observations of a sample of 48 Atacama Large Millimeter/ submillimeter Array detected submillimeter galaxies (SMGs) in the Extended Chandra Deep Field South field, to study their stellar morphologies and sizes. We detect $79 \% \pm 17 \%$ of the SMGs in the $H_{160}$-band imaging with a median sensitivity of $27.8 \mathrm{mag}$, and most $(80 \%)$ of the nondetections are SMGs with $870 \mu \mathrm{m}$ fluxes of $S_{870}<$ $3 \mathrm{mJy}$. With a surface brightness limit of $\mu_{H} \sim 26 \mathrm{mag} \mathrm{arcsec}^{-2}$, we find that $82 \% \pm 9 \%$ of the $H_{160}$-band-detected SMGs at $z=1-3$ appear to have disturbed morphologies, meaning they are visually classified as either irregulars or interacting systems, or both. By determining a Sérsic fit to the $H_{160}$ surface brightness profiles, we derive a median Sérsic index of $n=1.2 \pm 0.3$ and a median half-light radius of $r_{e}=4.4_{-0.5}^{+1.1} \mathrm{kpc}$ for our SMGs at $z=$ 1-3. We also find significant displacements between the positions of the $H_{160}$ component and $870 \mu \mathrm{m}$ emission in these systems, suggesting that the dusty starburst regions and less-obscured stellar distribution are not colocated. We find significant differences in the sizes and the Sérsic index between our $z=2-3$ SMGs and $z \sim 2$ quiescent galaxies, suggesting that a major transformation of the stellar light profile is needed in the quenching processes if SMGs are progenitors of the red-and-dead $z \sim 2$ galaxies. Given the short-lived nature of SMGs, we postulate that the majority of the $z=2-3$ SMGs with $S_{870} \gtrsim 2 \mathrm{mJy}$ are early/mid-stage major mergers.
\end{abstract}

Key words: cosmology: observations - galaxies: formation - galaxies: starburst - submillimeter: galaxies

\section{INTRODUCTION}

Bright submillimeter galaxies (SMGs) with $S_{870} \gtrsim 2 \mathrm{mJy}$ represent a population of distant, dust-obscured galaxies that were most prevalent around $10 \mathrm{Gyr}$ ago $(z \sim 2$; e.g., Smail et al. 1997; Barger et al. 1998; Hughes et al. 1998; Chapman et al. 2005; Wardlow et al. 2011; Yun et al. 2012; Simpson et al. 2014). Through extensive, multiwavelength observations, it appears that SMGs have many characteristics similar to local ultraluminous infrared galaxies (ULIRGs), such as their gas fractions, far-infrared luminosities, and interstellar medium (ISM) properties (Tacconi et al. 2008; Danielson et al. 2011; Bothwell et al. 2013; Riechers et al. 2011; Casey et al. 2014). With implied star formation rates (SFRs) of $\sim 500 M_{\odot} \mathrm{yr}^{-1}$, SMGs have the potential to form half of the stars in a massive galaxy $\left(\mathrm{M}_{\star}>10^{11} M_{\odot}\right)$ in just $\sim 100 \mathrm{Myr}$. Moreover, over the redshift range $z=1-4$, bright SMGs contribute $\sim 10 \%-30 \%$ of the total star formation budget (e.g., Barger et al. 2012; Casey et al. 2013; Swinbank et al. 2014). Inevitably, links have therefore been made between SMGs and passive elliptical galaxies (e.g., Lilly et al. 1999; Fu et al. 2013; Simpson et al. 2014). SMGs have also been linked to active galactic nucleus
(AGN)/QSO activity at $z \sim 2$ (e.g., Alexander et al. 2005; Coppin et al. 2008; Simpson et al. 2012; Wang et al. 2013) and the formation of the compact quiescent galaxy population found at $z=1-2$ (e.g., Cimatti et al. 2008; Whitaker et al. 2012; Toft et al. 2014).

However, there are also important differences between SMGs and local ULIRGs. In particular, the spatial extent of the gas and star formation in SMGs appears to be much larger than that typically seen in local ULIRGs (approximately a few kiloparsecs in SMGs, compared to just hundreds of parsecs in local ULIRGs; e.g., Charmandaris et al. 2002; Sakamoto et al. 2008; Menéndez-Delmestre et al. 2009; Díaz-Santos et al. 2010; Engel et al. 2010; Ivison et al. 2011; Rujopakarn et al. 2011). This could be due to the star formation occurring either in extended disks in high-redshift, pre-coalescence mergers or in tidal features associated with a late-stage merger. One route to testing the link between local ULIRGs and highredshift SMGs is by comparison to galaxy formation models. However, theoretical models attempting to reproduce the basic properties of bright SMGs (such as the number counts and redshift distribution) have come to a variety of conclusions regarding the physical processes that trigger the star formation: 
low-mass merging starbursts, with unusually low mass-to-light ratios (Baugh et al. 2005; Swinbank et al. 2008); isolated (or not strongly interacting), gas-rich disk galaxies with secular bursts (e.g., Davé et al. 2010; Cowley et al. 2014); and a hybrid scenario where some SMGs are merger-induced starbursts and some are secularly evolving disks (e.g., Hayward et al. 2011, 2013).

To distinguish chaotic systems such as mergers from ordered rotating disks, in principle we can compare the rotational velocity and velocity dispersions measured through emission lines originating from cool molecular gas and/or ionized gas, as well as searching for irregular morphologies, tidal features, or spiral arms in the stellar light in deep rest-frame optical/ near-infrared imaging. Many dynamical studies using emission lines from the ${ }^{12} \mathrm{CO}$ molecule or $\mathrm{H} \alpha$ have concluded that they see evidence of mergers (e.g., Tacconi et al. 2008; Engel et al. 2010; Alaghband-Zadeh et al. 2012; Menéndez-Delmestre et al. 2013). However, individual cases of clumpy, rotating disks have also been found (e.g., Swinbank et al. 2011; Hodge et al. 2012), although these do not preclude a merger origin (e.g., Robertson \& Bullock 2008; Ueda et al. 2014). Moreover, the drawback of studying kinematics based on molecular or ionized gas is that these tracers are sensitive to the dynamics of the ISM, in which the dense molecular gas could appear as rotating disks even in merging systems (e.g., NGC 3256; Sakamoto et al. 2006). Moreover, the dynamics of the ionized gas could be heavily affected by strong outflows (e.g., Harrison et al. 2012). A complementary approach is therefore to map the stellar distribution, the collisionless tracer of the galaxy morphology, to investigate the formation mechanism of bright SMGs.

Previous studies of the stellar morphologies of SMGs using high-resolution imaging from the Hubble Space Telescope (HST) have told a mixed story. The earliest optical HST studies of SMGs were confused by the counterpart identification problems that have plagued SMG samples (e.g., Smail et al. 1998). Using radio-located SMG samples with more robust counterpart identifications, Chapman et al. (2003) and Conselice et al. (2003) studied the morphologies of SMGs using rest-frame ultraviolet (UV) imaging. However, the rest-frame UV suffers strong obscuration in these very dusty sources, biasing the conclusions.

A key advance came with high-resolution near-infrared imaging from the $H S T$, which provides a less dust-sensitive probe of the stellar distribution on 0.'1 (subkiloparsec) scales. Such analyses with NICMOS have shown that SMGs have apparently compact and disturbed morphologies, with evidence for tidal tails and highly asymmetric light distributions (Swinbank et al. 2010; Conselice et al. 2011; Aguirre et al. 2013). However, at $z>1$ star-forming galaxies are often disturbed (e.g., Bluck et al. 2012); thus, such traits do not necessarily indicate an ongoing merger, as would be the case locally (e.g., Mortlock et al. 2013). Unfortunately, the faintness of SMG counterparts, combined with the relatively shallow surface brightness limits of the NICMOS imaging $\left(1 \sigma \mu_{H} \sim 24.5 \mathrm{mag} \operatorname{arcsec}^{-2}\right.$ ), meant that these studies could not differentiate the properties of the SMGs from those of relatively quiescent UV-selected galaxies at similar redshifts (Giavalisco 2002; Lotz et al. 2008; Law et al. 2012).

More recent studies of the stellar morphology of SMGs have used deeper $H_{160}$-band imaging from the new Wide Field Camera 3 (WFC3) on the HST with a substantially deeper surface brightness sensitivity, $\mu_{H} \sim 26 \mathrm{mag}_{\operatorname{arcsec}}{ }^{-2}$. Various quantitative analyses have been conducted, including the concentration $(C)$, asymmetry $(A)$, clumpiness $(S)$ (CAS) method (Conselice
2003), the Gini/M20 parameters, and fitting the light profile to determine the Sérsic indices and effective radius. In these studies, SMGs are found to have an average Sérsic index of $n \sim 1$, analogous to the local disky galaxies (Targett et al. 2013). Wiklind et al. (2014) also argue that SMGs represent a more isolated, asymmetric, and heterogeneous population in contrast with those dusty sources at similar redshifts but selected at 100/160 $\mu \mathrm{m}$ (Kartaltepe et al. 2012).

Unfortunately, even some of these recent morphological studies rely on samples of candidate SMGs selected from coarse resolution $\left(\sim 20^{\prime \prime}\right)$ of single-dish submillimeter surveys, which are identified through indirect, empirical correlations between the submillimeter emission and that at other wavelengths where higher angular resolution observations are possible, such as the radio or mid-infrared (MIR; e.g., Ivison et al. 1998, 2002; Smail et al. 2000; Pope et al. 2005; Biggs et al. 2011). While the success rate of these indirect techniques is upto $80 \%$, they suffer both contamination from false identifications and various selection biases (e.g., Wang et al. 2011; Hodge et al. 2013).

The only secure method for precisely locating the SMGs from a single-dish continuum survey is interferometric observations in the same waveband as the original discovery survey. The first interferometric studies of SMGs in the sub/millimeter were undertaken soon after their discovery, but they are observationally expensive and were initially limited to small samples (Frayer et al. 2000; Gear et al. 2000; Lutz et al. 2001; Dannerbauer et al. 2002, 2008; Iono et al. 2006; Wang et al. 2007, 2011; Younger et al. 2007, 2008, 2009; Cowie et al. 2009; Aravena et al. 2010; Hatsukade et al. 2010; Chen et al. 2011, 2014; Barger et al. 2012; Smolčić et al. 2012a, 2012b). In addition, some interferometric millimeter line surveys have also been undertaken both to locate the gas reservoir associated with the strongly star-forming SMGs and to simultaneously confirm their redshifts (e.g., Frayer et al. 1998; Greve et al. 2005; Bothwell et al. 2013), but these have typically targeted emission in low- $J{ }^{12} \mathrm{CO}$ transitions and hence may not unambiguously identify the precise location of the dust continuum counterparts (e.g., Tacconi et al. 2006; Ivison et al. 2010).

We have undertaken an Atacama Large Millimeter/ submillimeter Array (ALMA) Cycle 0, band 7 (870 $\mu \mathrm{m})$ study of the first large sample of submillimeter sources, a flux-limited sample of 126 submillimeter sources in the $0.5 \times 0.5$ Extended Chandra Deep Field South (ECDFS), taken from the LABOCA submillimeter single-dish survey at $870 \mu \mathrm{m}$ in ECDFS ("LESS" survey; Weiß et al. 2009). These ALMA maps directly pinpoint the position of each SMG to subarcsecond accuracy, free from the uncertainties due to the use of probabilistic radio/ MIR associations (Karim et al. 2013; Hodge et al. 2013). To study the stellar morphologies, we have collected a set of deep HST WFC3 $H_{160}$-band imaging on 48 of our ALMA study of the LESS sources (ALESS) SMGs with $1 \sigma$ depths of $\mu_{H} \sim 26 \mathrm{mag} \operatorname{arcsec}^{-2}, \sim 3$ times deeper than the surface brightness limits of previous NICMOS studies. The unambiguous identifications from the ALMA observations of a uniformly selected sample of SMGs, in combination with the improved near-infrared imaging capability of WFC3 on board the HST, now put us in a position to readdress the question of the morphological properties of SMGs and to test the theoretical models that predict different triggering mechanisms.

We describe the data and our methodology in Section 2. The results are given in Section 4. We discuss the implications of our results in Section 5 and summarize this paper in Section 6. Throughout this paper we adopt the $\mathrm{AB}$ magnitude system 
(Oke \& Gunn 1983), and we assume the Wilkinson Microwave Anisotropy Probe cosmology: $\mathrm{H}_{0}=70.5 \mathrm{~km} \mathrm{~s}^{-1} \mathrm{Mpc}^{-1}$, $\Omega_{M}=0.27$, and $\Omega_{\Lambda}=0.73$ (Larson et al. 2011).

\section{SAMPLE, OBSERVATIONS, AND DATA REDUCTION}

Our sample is drawn from the ALESS, which provide the first large-scale, unbiased identifications for a complete sample of SMGs. The full ALESS sample was presented in Hodge et al. (2013), which defined a robust "MAIN" catalog of 99 SMGs that are (1) located within the primary beam of ALMA, (2) detected with $\mathrm{S} / \mathrm{N} \geqslant 3.5$, (3) detected in the ALMA maps with a $1 \sigma \mathrm{rms}$ of less than $0.6 \mathrm{mJy}_{\text {beam }}{ }^{-1}$, and (4) have a major-tominor axis ratio of the synthesized beam of $\leqslant 2$. In addition, they also provided a "SUPPLEMENTARY" catalog of 32 SMGs that meet part of the criteria for the MAIN sample (i.e., $S / N \geqslant 4$ and have an rms $>0.6 \mathrm{mJy}_{\text {beam }^{-1}}$, lie outside the primary beam, or have a major-to-minor axis ratio $>2$ ).

\subsection{Hubble Space Telescope Observations}

The new HST/WFC3 observations were carried out in the $H_{160}$ band during Cycle 20 from late 2012 to mid-2013 (PID: 12866; PI: A. M. Swinbank) under LOW-SKY conditions in all exposures to minimize the background due to zodiacal light and Earth shine in order to probe the structural properties of any low surface brightness features. There were 15 pointings, and the exposure time was two orbits per pointing. The data were processed through the standard pipeline reduction using AstroDrizzLE. We also made use of images taken as part of two HST legacy programs, the Cosmic Assembly Near-infrared Deep Extragalactic Legacy Survey (CANDELS; Grogin et al. 2011; Koekemoer et al. 2011) and the Hubble Ultra Deep Field 2009 (HUDF09) program (Bouwens et al. 2011). The images taken by both programs have either similar or deeper depths in the $H_{160}$ band compared with our new data.

To calibrate the astrometry of our HST images, we aligned the $H_{160}$-band images to the $3.6 \mu \mathrm{m}$ image of the full ECDFS from Spitzer/IRAC, the common astrometric frame adopted in Simpson et al. (2014). We used SExTRACTOR to create a source catalog for each $H S T$ image and the $3.6 \mu \mathrm{m}$ imaging. We match each source in the HST catalog to the $3.6 \mu \mathrm{m}$ catalog and measure an individual offset for each image. We apply a median offset of $\Delta$ R.A. $=00^{\prime \prime} 11$ and $\Delta$ decl. $=-0^{\prime \prime} .25$, and all applied offsets are $<1^{\prime \prime}$. We statistically tested the accuracy of our astrometry by calculating the offsets between randomly chosen $3.6 \mu \mathrm{m}$ sources and their $H_{160}$-band counterparts lying within a 0.8 radius circle (equivalent to the IRAC $/ 3.6 \mu \mathrm{m}$ pointspread function (PSF)), and we found no systematic offset and a scatter of $\sim 0^{\prime} .16$ in both R.A. and decl., consistent with the expected accuracy of the IRAC imaging (Damen et al. 2011).

We used SExTRACTOR (Bertin \& Arnouts 1996) to extract sources from our HST imaging and set the detection threshold to be 16 connecting $1 \sigma$ pixels. We adopted the automatic aperture magnitudes (MAG_AUTO) for the flux measurements. Our SExTRACTOR settings on the detection threshold have yielded a range of $H_{160}$-band sensitivity between 27 and 30 mag with a median sensitivity of $\sim 27.8$ mag. The sensitivity was estimated by summing the background rms values assigned to each pixel by SEXTRACTOR, which are the rms values SEXTRACTOR used to trigger detections, within the $4 \times$ 4 pixel box (our detection threshold of 16 connecting $1 \sigma$ pixels) centered at the ALMA position.

\subsection{Our Sample}

To make efficient use of telescope time, we selected pointings for which there are two (or more) SMGs covered by the WFC3 field of view. We do not expect this to bias our results, as the photometric (and spectroscopic) redshifts of the SMGs in each WFC3 field do not suggest that the sources are physically associated. With 15 pointings, we have covered 48 ALESS SMGs. When combined with the 10 ALESS SMGs covered by CANDELS and the two ALESS SMGs observed by HUDF09, this brings the total sample to 60, in which 48 (12) sources are from the MAIN (SUPPLEMENTARY) catalog. In this study, our conclusions are drawn from the 48 ALESS SMGs in the MAIN catalog, a reliable catalog with $\sim 99 \%$ completeness and a false detection rate of $\sim 1.6 \%$ (Karim et al. 2013). In addition, we also present the results for the ALESS SMGs in the SUPPLEMENTARY catalog in Figures 10-13 and Tables 1 and 2. Note that two other ALESS SMGs were also covered by our HST observations, ALESS 55.5 and ALESS 67.2. However, in our $H_{160}$-band imaging, both appear to be physical associations of the same SMG; ALESS 55.5 is an extended clump eastward of ALESS 55.1, and ALESS 67.2 appears to be a separated detection $\sim 2$ " westward of ALESS 67.1. We therefore treated the two pairs, ALESS 55.1/55.5 and ALESS 67.1/67.2, as single sources in our analysis.

In Figure 1 we show example thumbnails of 20 ALESS SMGs covered by CANDELS/HUDF09. Our sample size is at least a factor of two larger than any previous nearinfrared SMG morphological studies, as well as benefiting from unambiguous and unbiased interferometric submillimeter identifications (Swinbank et al. 2006, 2010; Aguirre et al. 2013; Targett et al. 2013; Wiklind et al. 2014).

The histograms of $870 \mu \mathrm{m}$ fluxes and redshifts of the $48 \mathrm{H}_{160^{-}}$ band observed ALESS SMGs are shown in Figure 2, along with those of the ALESS parent population. A Kolmogorov-Smirnov (K-S) test shows that the $H S T$-observed subsample is drawn from the ALESS parent sample with a confidence level of $>99.5 \%$. For these tests we summed the $870 \mu \mathrm{m}$ fluxes of ALESS 55.1/55.5 and ALESS 67.1/67.2 and adopted the redshifts of ALESS 55.1 and ALESS 67.1 for the two pairs.

The photometric redshifts $\left(z_{\text {photo }}\right)$ of the ALESS SMGs were derived using the spectral energy distribution fitting code HYPERZ (Bolzonella et al. 2000) using the observed photometry from UV to MIR wavelengths (for details see Simpson et al. 2014). In total, 39 out of $48 H_{160}$-band observed ALESS SMGs have well-constrained photometric redshifts. This is confirmed by a comparison to the spectroscopic redshifts from A. L. R. Danielson et al. (in preparation), who find a median $\Delta z /\left(1+z_{\text {spec }}\right)=-0.004 \pm 0.026$. Together with the photometric redshifts, we used the $250-870 \mu \mathrm{m}$ photometry from Swinbank et al. (2014) to derive infrared luminosities $\left(L_{\mathrm{IR}}\right)$ for those $39 H_{160}$-band observed ALESS submillimeter galaxies (SMGs; Swinbank et al. 2014).

\subsection{Non-SMG Comparison Sample}

To provide a control sample to compare to the SMGs, we also analyzed a sample of field galaxies in an identical manner to the ALESS SMGs. We exploit the Multi-wavelength Survey by Yale-Chile (MUSYC) catalog, in which 32 band optical to MIR photometric measurements are provided along with the derived photometric redshifts (Cardamone et al. 2010). To define our sample, we selected any galaxies in the MUSYC catalog that are located within the ALMA primary beam but undetected in 
Table 1

GALFIT Results on MAIN ALESS SMGs

\begin{tabular}{|c|c|c|c|c|c|c|c|c|c|c|c|}
\hline $\begin{array}{l}\text { ALESS ID } \\
\text { (1) }\end{array}$ & $\begin{array}{c}\text { R.A. } \\
\text { (deg) } \\
(2)\end{array}$ & $\begin{array}{c}\text { Decl. } \\
\text { (deg) } \\
(3)\end{array}$ & $\begin{array}{c}z_{\text {photo }} \\
\text { (4) }\end{array}$ & $\begin{array}{c}H_{160} \\
\text { (ABmag) } \\
(5)\end{array}$ & $\begin{array}{l}\text { HID } \\
(6)\end{array}$ & $\begin{array}{c}\Delta R . A . \\
\left({ }^{\prime \prime}\right) \\
(7)\end{array}$ & $\begin{array}{c}\Delta \text { Decl. } \\
(") \\
(8)\end{array}$ & $\begin{array}{c}H_{160 g} \\
\text { (ABmag) } \\
(9)\end{array}$ & $\begin{array}{c}r_{e} \\
(\mathrm{kpc}) \\
(10)\end{array}$ & (11) & $\begin{array}{l}\chi_{v}^{2} \\
(12)\end{array}$ \\
\hline ALESS 001.1 & 53.31027 & -27.93737 & $4.34_{-1.43}^{+2.66}$ & $>27.8$ & $\cdots$ & $\cdots$ & $\cdots$ & $\cdots$ & $\cdots$ & $\cdots$ & $\cdots$ \\
\hline ALESS 001.2 & 53.31006 & -27.93656 & $4.64_{-1.02}^{+2.34}$ & $26.0 \pm 0.7$ & $\mathrm{H} 1$ & -0.72 & 0.13 & $26.50 \pm 0.24$ & $1.87_{-0.68}^{+0.61}$ & $0.96 \pm 1.41$ & 1.09 \\
\hline ALESS 001.3 & 53.30907 & -27.93676 & $2.84_{-0.30}^{+0.20}$ & $26.0 \pm 0.7$ & H1 & -0.25 & 0.01 & $25.69 \pm 0.29$ & $3.34_{-1.89}^{+1.89}$ & $3.73 \pm 2.59$ & 0.68 \\
\hline ALESS 002.1 & 53.26119 & -27.94521 & $1.96_{-0.20}^{+0.27}$ & $24.0 \pm 0.3$ & $\mathrm{H} 1$ & 0.18 & -0.07 & $23.98 \pm 0.02$ & $1.49_{-0.05}^{+0.05}$ & $1.33 \pm 0.13$ & 1.01 \\
\hline ALESS 002.2 & 53.26280 & -27.94525 & $\ldots$ & $26.4 \pm 0.9$ & $\mathrm{H} 1$ & -0.20 & -0.19 & $26.25 \pm 0.28$ & $\ldots$ & $1.56 \pm 1.29$ & 0.81 \\
\hline ALESS 003.1 & 53.33960 & -27.92230 & $3.90_{-0.59}^{+0.50}$ & $24.7 \pm 0.6$ & H1 & 0.25 & -0.48 & $24.67 \pm 0.07$ & $5.51_{-0.65}^{+0.68}$ & 1.00 & 1.06 \\
\hline ALESS 005.1 & 52.87047 & -27.98584 & $2.86_{-0.04}^{+0.05}$ & $25.6 \pm 0.7$ & $\cdots$ & $\cdots$ & $\ldots$ & $\ldots$ & $\ldots$ & $\cdots$ & $\cdots$ \\
\hline ALESS 009.1 & 53.04724 & -27.86998 & $4.50_{-2.33}^{+0.54}$ & $25.5 \pm 0.6$ & $\mathrm{H} 1$ & 0.19 & -0.80 & $25.06 \pm 0.30$ & $5.82_{-2.38}^{+2.77}$ & $1.29 \pm 0.77$ & 0.97 \\
\hline \multirow[t]{2}{*}{ ALESS 010.1} & 53.07942 & -27.87078 & $2.02_{-0.09}^{+0.09}$ & $24.0 \pm 0.4$ & $\mathrm{H} 1$ & -0.26 & -0.25 & $24.82 \pm 0.02$ & $1.18_{-0.05}^{+0.05}$ & $1.99 \pm 0.33$ & 0.88 \\
\hline & & & & & $\mathrm{H} 2$ & -0.33 & 0.60 & $24.26 \pm 0.03$ & $2.69_{-0.12}^{+0.12}$ & $1.67 \pm 0.14$ & 0.88 \\
\hline ALESS 013.1 & 53.20413 & -27.71439 & $3.25_{-0.46}^{+0.64}$ & $24.8 \pm 0.4$ & $\mathrm{H} 1$ & -1.52 & -0.24 & $24.54 \pm 0.56$ & $2.10_{-2.14}^{+2.14}$ & $3.88 \pm 3.85$ & 1.28 \\
\hline \multirow[t]{4}{*}{ ALESS 015.1} & 53.38903 & -27.99155 & $1.92_{-0.33}^{+0.62}$ & $23.8 \pm 0.5$ & $\mathrm{H} 1$ & 0.18 & -0.02 & $26.04 \pm 0.11$ & $1.95_{-0.20}^{+0.18}$ & $0.33 \pm 0.15$ & 0.84 \\
\hline & & & & & $\mathrm{H} 2$ & -0.41 & -0.18 & $24.47 \pm 0.09$ & $7.62_{-0.94}^{+0.89}$ & $1.06 \pm 0.16$ & 0.84 \\
\hline & & & & & H3 & 0.90 & -0.09 & $25.97 \pm 0.06$ & $2.38_{-0.22}^{+0.19}$ & $0.25 \pm 0.15$ & 0.84 \\
\hline & & & & & $\mathrm{H} 4$ & 0.54 & -0.96 & $26.16 \pm 0.08$ & $3.35_{-0.32}^{+0.30}$ & $0.20 \pm 0.13$ & 0.84 \\
\hline ALESS 015.3 & 53.38998 & -27.99318 & $\ldots$ & $>27.8$ & $\cdots$ & $\cdots$ & $\ldots$ & $\ldots$ & $\ldots$ & $\ldots$ & $\cdots$ \\
\hline \multirow[t]{2}{*}{ ALESS $017.1^{\mathrm{a}}$} & 53.03041 & -27.85576 & $1.50_{-0.07}^{+0.10}$ & $21.4 \pm 0.1$ & H1 & 0.42 & 0.19 & $22.04 \pm 0.04$ & $5.48_{-0.10}^{+0.10}$ & $0.28 \pm 0.01$ & 3.00 \\
\hline & & & & & $\mathrm{H} 2$ & 0.43 & 0.27 & $22.10 \pm 0.04$ & $1.28_{-0.05}^{+0.05}$ & $1.51 \pm 0.11$ & 3.00 \\
\hline \multirow[t]{2}{*}{ ALESS 018.1} & 53.02034 & -27.77993 & $2.04_{-0.06}^{+0.10}$ & $22.2 \pm 0.2$ & $\mathrm{H} 1$ & -0.08 & 0.12 & $22.06 \pm 0.01$ & $6.25_{-0.05}^{+0.04}$ & $0.32 \pm 0.01$ & 2.98 \\
\hline & & & & & $\mathrm{H} 2$ & -1.60 & 0.29 & $26.19 \pm 0.07$ & $1.11_{-0.26}^{+0.26}$ & $0.25 \pm 1.08$ & 2.98 \\
\hline \multirow[t]{2}{*}{ ALESS 029.1} & 53.40375 & -27.96926 & $2.66_{-0.76}^{+2.94}$ & $24.4 \pm 0.5$ & $\mathrm{H} 1$ & 0.24 & 0.38 & $25.50 \pm 0.06$ & $2.88_{-0.76}^{+0.27}$ & $0.42 \pm 0.13$ & 0.84 \\
\hline & & & & & $\mathrm{H} 2$ & -0.38 & -0.47 & $24.72 \pm 0.33$ & $6.79_{-3.85}^{+3.47}$ & $2.79 \pm 1.12$ & 0.84 \\
\hline \multirow[t]{2}{*}{ ALESS 039.1} & 52.93763 & -27.57687 & $2.44_{-0.23}^{+0.18}$ & $23.5 \pm 0.2$ & H1 & 0.03 & -0.08 & $24.78 \pm 0.08$ & $2.42_{-0.08}^{+0.09}$ & $0.10 \pm 0.06$ & 0.91 \\
\hline & & & & & $\mathrm{H} 2$ & -0.49 & 0.05 & $23.33 \pm 0.34$ & $12.09_{-5.40}^{+5.41}$ & $2.10 \pm 0.67$ & 0.91 \\
\hline \multirow[t]{2}{*}{ ALESS 043.1} & 53.27767 & -27.80068 & $1.70_{-0.12}^{+0.20}$ & $23.3 \pm 0.3$ & $\mathrm{H} 1$ & 0.01 & 0.05 & $23.40 \pm 0.03$ & $8.60_{-0.35}^{+0.35}$ & $0.94 \pm 0.06$ & 1.29 \\
\hline & & & & & $\mathrm{H} 2$ & 0.21 & -0.94 & $25.11 \pm 0.42$ & $6.71_{-3.68}^{+3.68}$ & $1.68 \pm 0.91$ & 1.29 \\
\hline \multirow[t]{2}{*}{ ALESS $045.1^{\mathrm{a}}$} & 53.10526 & -27.87515 & $2.34_{-0.67}^{+0.26}$ & $23.6 \pm 0.3$ & $\mathrm{H} 1$ & -0.11 & -0.06 & $23.89 \pm 0.09$ & $6.15_{-0.76}^{+0.77}$ & 4.00 & 1.02 \\
\hline & & & & & $\mathrm{H} 2$ & 0.14 & -0.36 & $24.83 \pm 0.04$ & $3.27_{-0.17}^{+0.19}$ & $0.52 \pm 0.10$ & 1.02 \\
\hline \multirow[t]{2}{*}{ ALESS 049.1} & 52.85300 & -27.84641 & $2.76_{-0.14}^{+0.11}$ & $23.1 \pm 0.2$ & $\mathrm{H} 1$ & -0.11 & 0.40 & $24.76 \pm 0.09$ & $5.25_{-0.53}^{+0.53}$ & 1.00 & 1.67 \\
\hline & & & & & $\mathrm{H} 2$ & -0.42 & 0.30 & $22.91 \pm 0.09$ & $4.43_{-0.80}^{+0.80}$ & $4.90 \pm 0.63$ & 1.67 \\
\hline \multirow[t]{2}{*}{ ALESS 049.2} & 52.85196 & -27.84391 & $1.46_{-0.10}^{+0.07}$ & $23.1 \pm 0.2$ & $\mathrm{H} 1$ & -0.05 & -0.20 & $23.60 \pm 0.06$ & $3.16_{-0.17}^{+0.17}$ & $1.24 \pm 0.10$ & 1.51 \\
\hline & & & & & $\mathrm{H} 2$ & 0.17 & 0.38 & $24.16 \pm 0.11$ & $2.90_{-0.19}^{+0.19}$ & $0.18 \pm 0.04$ & 1.51 \\
\hline \multirow[t]{3}{*}{ ALESS 051.1} & 52.93775 & -27.74092 & $1.22_{-0.06}^{+0.03}$ & $19.6 \pm 0.1$ & $\mathrm{H} 1$ & 0.54 & 0.16 & $21.53 \pm 0.03$ & $3.93_{-0.20}^{+0.19}$ & 4.00 & 21.06 \\
\hline & & & & & $\mathrm{H} 2$ & 1.40 & 1.14 & $24.66 \pm 0.25$ & $2.81_{-0.88}^{+0.88}$ & $0.84 \pm 0.73$ & 21.06 \\
\hline & & & & & $\mathrm{H} 3$ & -1.45 & -1.86 & $19.70 \pm 0.01$ & $4.86_{-0.08}^{+0.07}$ & $1.88 \pm 0.03$ & 21.06 \\
\hline ALESS 055.1 & 53.25924 & -27.67651 & $2.05_{-0.13}^{+0.15}$ & $23.2 \pm 0.3$ & H1 & 0.43 & -0.39 & $23.06 \pm 0.01$ & $3.32_{-0.06}^{+0.06}$ & $1.09 \pm 0.03$ & 8.66 \\
\hline ALESS 055.2 & 53.25898 & -27.67815 & $\ldots$ & $>30.3$ & $\cdots$ & $\cdots$ & $\cdots$ & $\ldots$ & $\ldots$ & $\ldots$ & $\ldots$ \\
\hline \multirow[t]{2}{*}{ ALESS $057.1^{\mathrm{a}}$} & 52.96635 & -27.89085 & $2.95_{-0.09}^{+0.05}$ & $23.3 \pm 0.2$ & $\mathrm{H} 1$ & 0.14 & 0.09 & $23.53 \pm 0.04$ & $2.21_{-0.10}^{+0.11}$ & $1.75 \pm 0.13$ & 1.34 \\
\hline & & & & & $\mathrm{H} 2$ & 0.25 & -0.39 & $24.60 \pm 0.12$ & $3.23_{-0.46}^{+0.46}$ & $1.30 \pm 0.28$ & 1.34 \\
\hline \multirow[t]{4}{*}{ ALESS $067.1^{\mathrm{a}}$} & 53.17998 & -27.92065 & $2.13_{-0.09}^{+0.05}$ & $21.7 \pm 0.2$ & H1 & 0.10 & -0.26 & $22.14 \pm 0.03$ & $9.55_{-0.39}^{+0.39}$ & $1.42 \pm 0.06$ & 1.45 \\
\hline & & & & & $\mathrm{H} 2$ & -0.51 & -0.22 & $24.22 \pm 0.02$ & $1.61_{-0.04}^{+0.04}$ & $0.35 \pm 0.08$ & 1.45 \\
\hline & & & & & $\mathrm{H} 3$ & -0.11 & -0.68 & $24.42 \pm 0.02$ & $1.13_{-0.04}^{+0.04}$ & $0.40 \pm 0.20$ & 1.45 \\
\hline & & & & & $\mathrm{H} 4$ & 0.96 & -0.99 & $23.57 \pm 0.01$ & $1.64_{-0.03}^{+0.03}$ & $0.77 \pm 0.05$ & 1.45 \\
\hline ALESS 069.1 & 52.89073 & -27.99234 & $2.34_{-0.44}^{+0.27}$ & $24.1 \pm 0.3$ & H1 & 0.06 & 0.06 & $24.00 \pm 0.02$ & $4.13_{-0.16}^{+0.17}$ & $0.54 \pm 0.05$ & 1.00 \\
\hline ALESS 069.2 & 52.89223 & -27.99136 & $\ldots$ & $>27.9$ & $\cdots$ & $\cdots$ & $\cdots$ & $\ldots$ & $\ldots$ & $\ldots$ & $\cdots$ \\
\hline ALESS 069.3 & 52.89152 & -27.99398 & $\ldots$ & $>27.9$ & $\cdots$ & $\cdots$ & $\cdots$ & $\cdots$ & $\cdots$ & $\cdots$ & $\cdots$ \\
\hline ALESS $073.1^{\mathrm{a}}$ & 53.12205 & -27.93881 & $5.18_{-0.45}^{+0.43}$ & $24.0 \pm 0.3$ & $\mathrm{H} 1$ & 0.03 & 0.01 & $23.93 \pm 0.01$ & $<1.19$ & $\cdots$ & 2.02 \\
\hline \multirow[t]{2}{*}{ ALESS 074.1} & 53.28811 & -27.80477 & $1.80_{-0.13}^{+0.13}$ & $23.4 \pm 0.2$ & H1 & 0.02 & 0.37 & $23.42 \pm 0.05$ & $5.69_{-0.41}^{+0.41}$ & $1.65 \pm 0.12$ & 0.94 \\
\hline & & & & & $\mathrm{H} 2$ & -0.19 & -0.37 & $25.05 \pm 0.06$ & $2.42_{-0.13}^{+0.13}$ & $0.39 \pm 0.09$ & 0.94 \\
\hline ALESS 079.1 & 53.08806 & -27.94083 & $2.04_{-0.31}^{+0.63}$ & $24.6 \pm 0.5$ & $\mathrm{H} 1$ & -0.10 & -0.06 & $23.95 \pm 0.05$ & $7.58_{-0.67}^{+0.58}$ & 1.00 & 1.17 \\
\hline
\end{tabular}


Table 1

(Continued)

\begin{tabular}{|c|c|c|c|c|c|c|c|c|c|c|c|}
\hline $\begin{array}{l}\text { ALESS ID } \\
\text { (1) }\end{array}$ & $\begin{array}{l}\text { R.A. } \\
\text { (deg) } \\
\text { (2) }\end{array}$ & $\begin{array}{l}\text { Decl. } \\
\text { (deg) } \\
\text { (3) }\end{array}$ & $\begin{array}{c}z_{\text {photo }} \\
\text { (4) }\end{array}$ & $\begin{array}{c}H_{160} \\
\text { (ABmag) } \\
(5)\end{array}$ & $\begin{array}{l}\text { HID } \\
(6)\end{array}$ & $\begin{array}{c}\Delta \text { R.A. } \\
\left({ }^{\prime \prime}\right) \\
(7)\end{array}$ & $\begin{array}{c}\Delta \text { Decl. } \\
(") \\
(8)\end{array}$ & $\begin{array}{c}H_{160 g} \\
(\mathrm{ABmag}) \\
(9)\end{array}$ & $\begin{array}{c}r_{e} \\
(\mathrm{kpc}) \\
(10)\end{array}$ & $\begin{array}{c}n \\
(11)\end{array}$ & $\chi_{v}^{2}$ \\
\hline \multirow[t]{4}{*}{ ALESS 079.2} & 53.09000 & -27.93999 & $1.55_{-0.18}^{+0.10}$ & $21.9 \pm 0.1$ & H1 & -0.22 & -0.24 & $23.20 \pm 0.03$ & $3.15_{-0.08}^{+0.07}$ & $0.30 \pm 0.03$ & 2.11 \\
\hline & & & & & $\mathrm{H} 2$ & -0.58 & -0.12 & $23.87 \pm 0.05$ & $1.29_{-0.05}^{+0.05}$ & $0.98 \pm 0.13$ & 2.11 \\
\hline & & & & & $\mathrm{H} 3$ & 0.11 & 0.63 & $22.32 \pm 0.06$ & $8.09_{-0.61}^{+0.61}$ & $1.88 \pm 0.13$ & 2.11 \\
\hline & & & & & $\mathrm{H} 4$ & -0.42 & -0.75 & $23.03 \pm 0.02$ & $4.29_{-0.08}^{+0.08}$ & $0.76 \pm 0.03$ & 2.11 \\
\hline ALESS 079.4 & 53.08826 & -27.94181 & $\ldots$ & $>28.7$ & $\cdots$ & $\cdots$ & $\ldots$ & $\ldots$ & $\ldots$ & $\ldots$ & $\cdots$ \\
\hline \multirow[t]{2}{*}{ ALESS 082.1} & 53.22499 & -27.63747 & $2.10_{-0.44}^{+3.27}$ & $24.0 \pm 0.4$ & H1 & -0.13 & -0.23 & $24.14 \pm 0.03$ & $4.19_{-1.14}^{+0.22}$ & $0.71 \pm 0.07$ & 1.18 \\
\hline & & & & & $\mathrm{H} 2$ & -1.25 & 0.38 & $25.88 \pm 0.13$ & $2.55_{-0.81}^{+0.44}$ & $1.21 \pm 0.64$ & 1.18 \\
\hline \multirow[t]{2}{*}{ ALESS 087.1} & 53.21202 & -27.52819 & $3.20_{-0.47}^{+0.08}$ & $22.5 \pm 0.1$ & $\mathrm{H} 1$ & -0.24 & -0.07 & $23.92 \pm 0.02$ & $1.03_{-0.03}^{+0.06}$ & $0.49 \pm 0.08$ & 1.95 \\
\hline & & & & & $\mathrm{H} 2$ & -0.74 & -0.30 & $21.97 \pm 0.13$ & $12.92_{-2.64}^{+2.70}$ & $3.88 \pm 0.43$ & 1.95 \\
\hline ALESS 087.3 & 53.21361 & -27.53075 & $\ldots$ & $>27.9$ & $\cdots$ & $\ldots$ & $\ldots$ & $\ldots$ & $\ldots$ & $\ldots$ & $\cdots$ \\
\hline \multirow[t]{6}{*}{ ALESS 088.1} & 52.97818 & -27.89486 & $1.84_{-0.11}^{+0.12}$ & $22.3 \pm 0.3$ & $\mathrm{H} 1$ & 0.04 & 0.32 & $24.48 \pm 0.05$ & $6.63_{-0.48}^{+0.48}$ & 1.00 & 1.26 \\
\hline & & & & & $\mathrm{H} 2$ & -0.38 & -0.34 & $23.57 \pm 0.01$ & $2.19_{-0.04}^{+0.04}$ & $0.86 \pm 0.06$ & 1.26 \\
\hline & & & & & $\mathrm{H} 3$ & -0.41 & 0.48 & $24.72 \pm 0.03$ & $4.60_{-0.16}^{+0.16}$ & $0.08 \pm 0.07$ & 1.26 \\
\hline & & & & & $\mathrm{H} 4$ & 0.75 & 0.13 & $23.77 \pm 0.11$ & $6.56_{-0.92}^{+0.92}$ & 4.00 & 1.26 \\
\hline & & & & & H5 & 1.57 & -0.06 & $23.47 \pm 0.25$ & $16.20_{-3.82}^{+3.82}$ & 4.00 & 1.26 \\
\hline & & & & & H6 & 1.61 & 0.30 & $24.57 \pm 0.09$ & $1.85_{-0.18}^{+0.18}$ & $1.57 \pm 0.36$ & 1.26 \\
\hline \multirow[t]{3}{*}{ ALESS 088.11} & 52.97895 & -27.89378 & $2.56_{-0.12}^{+0.04}$ & $23.2 \pm 0.2$ & $\mathrm{H} 1$ & -0.10 & 0.64 & $23.28 \pm 0.04$ & $1.65_{-0.12}^{+0.12}$ & $4.03 \pm 0.43$ & 1.22 \\
\hline & & & & & $\mathrm{H} 2$ & 0.06 & 1.12 & $25.78 \pm 0.40$ & $3.29_{-1.91}^{+1.91}$ & 4.00 & 1.22 \\
\hline & & & & & $\mathrm{H} 3$ & -0.26 & 1.36 & $25.11 \pm 0.14$ & $6.08_{-1.11}^{+1.12}$ & 1.00 & 1.22 \\
\hline ALESS 088.2 & 52.98080 & -27.89453 & $\ldots$ & $>27.9$ & $\cdots$ & $\ldots$ & $\cdots$ & $\ldots$ & $\ldots$ & $\cdots$ & $\cdots$ \\
\hline \multirow[t]{3}{*}{ ALESS 088.5} & 52.98252 & -27.89645 & $2.30_{-0.50}^{+0.11}$ & $23.1 \pm 0.2$ & $\mathrm{H} 1$ & 0.19 & 0.38 & $25.79 \pm 0.08$ & $1.85_{-0.22}^{+0.23}$ & $0.57 \pm 0.40$ & 1.49 \\
\hline & & & & & $\mathrm{H} 2$ & -0.28 & -0.41 & $23.67 \pm 0.05$ & $4.89_{-0.32}^{+0.34}$ & $1.06 \pm 0.10$ & 1.49 \\
\hline & & & & & $\mathrm{H} 3$ & -0.49 & -0.95 & $24.04 \pm 0.04$ & $1.84_{-0.06}^{+0.07}$ & $0.55 \pm 0.06$ & 1.49 \\
\hline \multirow[t]{2}{*}{ ALESS 092.2} & 52.90891 & -27.72872 & $1.90_{-0.75}^{+0.27}$ & $24.7 \pm 0.6$ & $\mathrm{H} 1$ & 0.48 & -0.40 & $25.85 \pm 0.05$ & $1.61_{-0.14}^{+0.00}$ & $0.24 \pm 0.16$ & 0.96 \\
\hline & & & & & $\mathrm{H} 2$ & -0.21 & 0.98 & $24.74 \pm 0.23$ & $3.59_{-1.17}^{+0.00}$ & $1.98 \pm 0.79$ & 0.96 \\
\hline ALESS 094.1 & 53.28164 & -27.96828 & $2.87_{-0.64}^{+0.37}$ & $24.1 \pm 0.3$ & H1 & 0.26 & -0.40 & $23.67 \pm 0.11$ & $7.92_{-1.02}^{+1.07}$ & 1.00 & 1.88 \\
\hline \multirow[t]{5}{*}{ ALESS 112.1} & 53.20360 & -27.52036 & $1.95_{-0.26}^{+0.15}$ & $22.2 \pm 0.2$ & H1 & -0.05 & 0.15 & $24.16 \pm 0.12$ & $0.87_{-0.03}^{+0.03}$ & $1.08 \pm 0.21$ & 1.31 \\
\hline & & & & & $\mathrm{H} 2$ & -0.18 & -0.02 & $23.81 \pm 0.05$ & $3.46_{-0.06}^{+0.06}$ & $0.16 \pm 0.02$ & 1.31 \\
\hline & & & & & $\mathrm{H} 3$ & 0.08 & 0.17 & $23.03 \pm 0.27$ & $7.32_{-4.46}^{+4.46}$ & $4.58 \pm 1.47$ & 1.31 \\
\hline & & & & & $\mathrm{H} 4$ & -0.59 & 0.37 & $23.48 \pm 0.31$ & $10.15_{-4.08}^{+4.08}$ & $2.22 \pm 0.59$ & 1.31 \\
\hline & & & & & H5 & 0.00 & 1.07 & $25.55 \pm 0.05$ & $1.88_{-0.15}^{+0.15}$ & $0.25 \pm 0.20$ & 1.31 \\
\hline ALESS 114.1 & 52.96036 & -27.74593 & $\ldots$ & $>27.9$ & $\cdots$ & $\cdots$ & $\cdots$ & $\ldots$ & $\ldots$ & $\ldots$ & $\cdots$ \\
\hline \multirow[t]{2}{*}{ ALESS $114.2^{\mathrm{a}}$} & 52.96294 & -27.74369 & $1.56_{-0.07}^{+0.07}$ & $21.1 \pm 0.1$ & $\mathrm{H} 1$ & -0.13 & -0.09 & $21.32 \pm 0.02$ & $3.12_{-0.06}^{+0.06}$ & $1.65 \pm 0.08$ & 2.48 \\
\hline & & & & & $\mathrm{H} 2$ & -0.05 & -0.04 & $22.40 \pm 0.05$ & $0.96_{-0.01}^{+0.01}$ & $0.17 \pm 0.06$ & 2.48 \\
\hline ALESS 116.1 & 52.97634 & -27.75804 & $3.54_{-0.87}^{+1.47}$ & $>28.0$ & $\cdots$ & $\cdots$ & $\cdots$ & $\cdots$ & $\ldots$ & $\ldots$ & $\cdots$ \\
\hline ALESS 116.2 & 52.97683 & -27.75874 & $4.01_{-2.19}^{+1.19}$ & $26.7 \pm 1.0$ & $\mathrm{H} 1$ & 0.15 & -0.05 & $25.84 \pm 0.22$ & $5.40_{-1.66}^{+1.91}$ & 1.00 & 0.64 \\
\hline ALESS 118.1 & 52.84135 & -27.82816 & $2.26_{-0.23}^{+0.50}$ & $24.3 \pm 0.3$ & $\mathrm{H} 1$ & -0.14 & -0.16 & $24.64 \pm 0.11$ & $2.06_{-0.34}^{+0.34}$ & $2.10 \pm 0.56$ & 1.38 \\
\hline
\end{tabular}

Notes. In Columns 1-3 we give the source IDs and the sky positions in right ascension (R.A.) and declination (decl.), both J2000, adopted from Hodge et al. (2013). In Column 4, we give the photometric redshifts derived by Simpson et al. (2014), in which the errors show the $1 \sigma$ uncertainty. In Column 5 we show the measured total $H_{160}$-band flux densities in AB magnitude using SExTrACTOR, in which the sensitivity limit is given for the nondetections. Note that the uncertainties of the $H_{160}$-band flux include Poisson errors based on our adopted CCD gain of 2.4 for WFC3/IR (Dressel 2014), and in our case the Poisson errors dominate over sky noise. In Column 6 we list the designated IDs for each component detected in our $H_{160}$-band imaging. In Columns $7-12$ we present the GALFIT results. In Columns 7 and 8 we show the displacements in sky positions in arcseconds between the $H_{160}$-band component and the ALMA $870 \mu$ m positions given in Columns 2 and 3 , in which the positive values in R.A. and decl. indicate displacements toward east and north with respect to the ALMA positions. The mean errors of displacements based on the model fit are 0.'012 \pm 0'.01 in R.A. and 0.'011 \pm 0.009 in decl. In Column 9 we give the $H_{160}$-band flux densities in AB magnitudes of the model Sérsic profile, with the effective radius $\left(r_{e}\right)$ and the Sérsic index $(n)$ given in Columns 10 and 11. The errors of $r_{e}$ include the errors of both the photometric redshifts and the model fit, and the Sérsic indices without errors are fixed values, 1.0 or 4.0, in the model fit (last paragraph of Section 3.2). The reduced chi-square ( $\chi_{v}^{2}$ ) values of the GALFIT Sérsic models are shown in Column 12. The $\chi_{v}^{2}$ values are the same for every component corresponding to the same SMG because GALFIT performed simultaneous fitting to the masked pixels.

${ }^{\text {a }}$ X-ray-identified AGNs in Wang et al. (2013). 
Table 2

GALFIT Results on SUPPLEMENTARY ALESS SMGs

\begin{tabular}{|c|c|c|c|c|c|c|c|c|c|c|c|}
\hline $\begin{array}{l}\text { ALESS ID } \\
\text { (1) }\end{array}$ & $\begin{array}{l}\text { R.A. } \\
\text { (deg) } \\
(2)\end{array}$ & $\begin{array}{c}\text { Decl. } \\
(\mathrm{deg}) \\
(3)\end{array}$ & $\begin{array}{c}z_{\text {photo }} \\
\text { (4) }\end{array}$ & $\begin{array}{c}H_{160} \\
(\mathrm{ABmag}) \\
(5)\end{array}$ & $\begin{array}{l}\text { HID } \\
\text { (6) }\end{array}$ & $\begin{array}{c}\Delta \mathrm{R} . \mathrm{A} . \\
\left({ }^{\prime \prime}\right) \\
(7)\end{array}$ & $\begin{array}{c}\Delta \text { Decl. } \\
\left({ }^{\prime \prime}\right) \\
(8)\end{array}$ & $\begin{array}{c}H_{160 g} \\
(\mathrm{ABmag}) \\
(9)\end{array}$ & $\begin{array}{c}R_{e} \\
(\mathrm{kpc}) \\
(10)\end{array}$ & $\begin{array}{c}n \\
(11)\end{array}$ & $\begin{array}{l}\chi^{2} \\
(12)\end{array}$ \\
\hline ALESS 003.2 & 53.34246 & -27.92249 & $1.39_{-0.34}^{+0.37}$ & $>27.8$ & $\cdots$ & $\cdots$ & $\cdots$ & $\cdots$ & $\cdots$ & $\cdots$ & $\cdots$ \\
\hline ALESS 003.3 & 53.33629 & -27.92056 & $\ldots$ & $>27.9$ & $\cdots$ & $\cdots$ & $\cdots$ & $\cdots$ & $\cdots$ & $\cdots$ & $\cdots$ \\
\hline ALESS 003.4 & 53.34164 & -27.91938 & $\ldots$ & $>27.9$ & $\ldots$ & $\cdots$ & $\ldots$ & $\ldots$ & $\ldots$ & $\cdots$ & $\ldots$ \\
\hline \multirow[t]{2}{*}{ ALESS 015.2} & 53.39188 & -27.99172 & $0.72_{-0.72}^{+0.65}$ & $25.6 \pm 0.9$ & $\mathrm{H} 1$ & -0.40 & 0.83 & $26.56 \pm 0.09$ & $1.43_{-1.44}^{+0.29}$ & $0.24 \pm 0.46$ & 0.91 \\
\hline & & & & & $\mathrm{H} 2$ & -0.88 & 1.40 & $26.02 \pm 0.08$ & $2.55_{-2.57}^{+0.51}$ & $0.35 \pm 0.16$ & 0.91 \\
\hline ALESS 015.6 & 53.38819 & -27.99505 & $\ldots$ & $>27.9$ & $\cdots$ & $\ldots$ & $\cdots$ & $\ldots$ & $\ldots$ & $\ldots$ & $\ldots$ \\
\hline ALESS 017.2 & 53.03444 & -27.85547 & $2.10_{-1.35}^{+0.62}$ & $25.1 \pm 0.7$ & $\mathrm{H} 1$ & -0.41 & -0.48 & $25.31 \pm 0.08$ & $4.04_{-0.79}^{+0.00}$ & 1.00 & 0.93 \\
\hline \multirow[t]{2}{*}{ ALESS 017.3} & 53.03072 & -27.85942 & $2.58_{-0.25}^{+0.14}$ & $24.3 \pm 0.5$ & $\mathrm{H} 1$ & 0.15 & 0.72 & $25.90 \pm 0.05$ & $1.13_{-0.11}^{+0.11}$ & $0.64 \pm 0.35$ & 0.71 \\
\hline & & & & & $\mathrm{H} 2$ & -0.53 & -0.66 & $24.50 \pm 0.02$ & $1.42_{-0.05}^{+0.05}$ & $0.87 \pm 0.12$ & 0.71 \\
\hline ALESS 034.1 & 53.07483 & -27.87591 & $1.86_{-0.32}^{+0.29}$ & $26.1 \pm 0.8$ & $\mathrm{H} 1$ & -0.23 & -0.49 & $26.48 \pm 0.04$ & $0.81_{-0.12}^{+0.12}$ & 1.00 & 0.63 \\
\hline \multirow[t]{2}{*}{ ALESS 038.1} & 53.29515 & -27.94450 & $2.47_{-0.05}^{+0.10}$ & $22.2 \pm 0.2$ & $\mathrm{H} 1$ & -0.34 & -0.37 & $24.00 \pm 0.03$ & $3.51_{-0.16}^{+0.15}$ & $0.89 \pm 0.07$ & 0.99 \\
\hline & & & & & $\mathrm{H} 2$ & 0.95 & 0.20 & $22.37 \pm 0.01$ & $3.05_{-0.04}^{+0.03}$ & $0.99 \pm 0.02$ & 0.99 \\
\hline ALESS 039.2 & 52.93567 & -27.57865 & $0.79_{-0.17}^{+0.28}$ & $>27.8$ & $\cdots$ & $\ldots$ & $\ldots$ & $\ldots$ & $\ldots$ & $\ldots$ & $\cdots$ \\
\hline ALESS 043.3 & 53.27612 & -27.79853 & $1.98_{-0.99}^{+0.88}$ & $>27.8$ & $\cdots$ & $\cdots$ & $\cdots$ & $\ldots$ & $\ldots$ & $\ldots$ & $\cdots$ \\
\hline \multirow[t]{5}{*}{ ALESS 101.1} & 52.96499 & -27.76472 & $3.49_{-0.88}^{+3.52}$ & $23.4 \pm 0.4$ & $\mathrm{H} 1$ & -0.10 & -0.19 & $24.50 \pm 0.22$ & $9.39_{-3.34}^{+2.18}$ & $1.08 \pm 0.22$ & 1.14 \\
\hline & & & & & $\mathrm{H} 2$ & 0.66 & -0.91 & $24.90 \pm 0.28$ & $4.50_{-2.50}^{+2.19}$ & $3.61 \pm 1.35$ & 1.14 \\
\hline & & & & & $\mathrm{H} 3$ & -1.14 & -0.84 & $24.99 \pm 0.03$ & $1.72_{-0.50}^{+0.17}$ & $0.52 \pm 0.09$ & 1.14 \\
\hline & & & & & $\mathrm{H} 4$ & -2.02 & -1.00 & $25.26 \pm 0.30$ & $7.08_{-3.53}^{+2.97}$ & 4.00 & 1.14 \\
\hline & & & & & $\mathrm{H} 5$ & -1.80 & -1.63 & $26.26 \pm 0.09$ & $1.38_{-0.46}^{+0.27}$ & $0.80 \pm 0.60$ & 1.14 \\
\hline
\end{tabular}

the ALMA imaging $\left(S_{870} \lesssim 2 \mathrm{mJy}\right)$. We only select sources that are located at $z=1-3$, the redshift range in which we focus our analysis. We adopted the spectroscopic redshifts if available, and for sources with only photometric redshifts we made a quality cut of $Q_{z}<2$, as suggested by the author of EAZY (the program used for deriving the photometric redshift) because the scatter increases sharply above $Q_{z}=2$ and the $5 \sigma$ outlier fraction on sources with $Q_{z}>2$ increases to around $30 \%$ (Brammer et al. 2008). Within these selection limits, there are 58 galaxies. We note that on average the comparison sample has a redshift distribution that is skewed to slightly lower redshift, with $\langle z\rangle=1.5_{-0.3}^{+0.9}$, than the SMGs.

\section{METHODOLOGY}

Studies of galaxy morphology often involve analysis using quantitative tools such as the CAS method (Conselice 2003), the Gini coefficient (Abraham et al. 2003), and the M20 parameter (Lotz et al. 2004). However, although quantitative methods are useful tools for building objective references and separating bulge-dominated early-type galaxies from disk-like late-type galaxies, on some occasions shapes or features that are obvious to the eye, and critical for separating irregulars/ mergers from disks, are hard to distinguish with these methods. For example, CAS analysis in the optical $R$-band imaging is virtually incapable of distinguishing between local late-type disks, irregulars, and edge-on disks (Conselice 2003), as the nonparametric method is only sensitive to rankings of the pixel value, not permutations of the pixel position. ${ }^{15}$ One can imagine

\footnotetext{
15 For example, the Gini coefficient is used to measure the distribution of light among pixels, with higher values of the coefficient indicating an unequal distribution (Gini of 1 means all light is located in one pixel), while lower values indicate more even distributions (Gini of 0 means every pixel has an even share). The Gini value is calculated using the Lorentz curve (Lorentz 1905) of the galaxies' light distribution, which does not take any spatial information into consideration. We refer the reader to Conselice (2014) for a full review on nonparametric methods.
}

a face-on disk with spiral arms having similar CAS values as a face-on irregular with the same pixel value distribution of that face-on disk but with pixel positions shuffled. Indeed, at $z>1$ late-type galaxies are shown to be often disturbed (Bluck et al. 2012), and CAS analysis cannot separate disks from irregulars (Mortlock et al. 2013), which could be the reason that previous morphological studies of SMGs found separating $z \sim 2 \mathrm{UV}$ selected galaxies from $z \sim 2$ SMGs difficult using Gini/M20 or CAS (Swinbank et al. 2010; Wiklind et al. 2014).

This caveat on nonparametric analysis motivates the need to first conduct a visual inspection of the morphology of SMGs before we measure the size and surface brightness profiles (e.g., van Dokkum et al. 2010). Hence, we next study our sample of ALESS SMGs using visual classifications, along with the surface brightness fitting tool, GALFIT, to measure effective radius (half-light radius) and Sérsic indices.

\subsection{Visual Classifications}

Following recent $H_{160}$-band morphological studies in the CANDELS fields (e.g., Kartaltepe et al. 2012; Kocevski et al. 2012), we visually classified the $H_{160}$-band morphologies of our HST-observed ALESS SMGs into five main morphological classes, which are described as follows.

1. Disk - sources with apparent disk-like structure, regardless of whether they display spiral arms, or having an elongated morphology with or without a central bulge.

2. Spheroid — sources with apparent circular/ellipsoidal structure with resolved, centrally concentrated emission.

3. Irregular-sources with structures that do not belong to any of the other classes, or sources that are classified as disks or spheroids but have disturbed structures in the outskirts (noted as disk+irregular or spheroid+irregular).

4. Unresolved-sources with structures that are well fit by a PSF (detailed model fits are described in Section 3.2). 


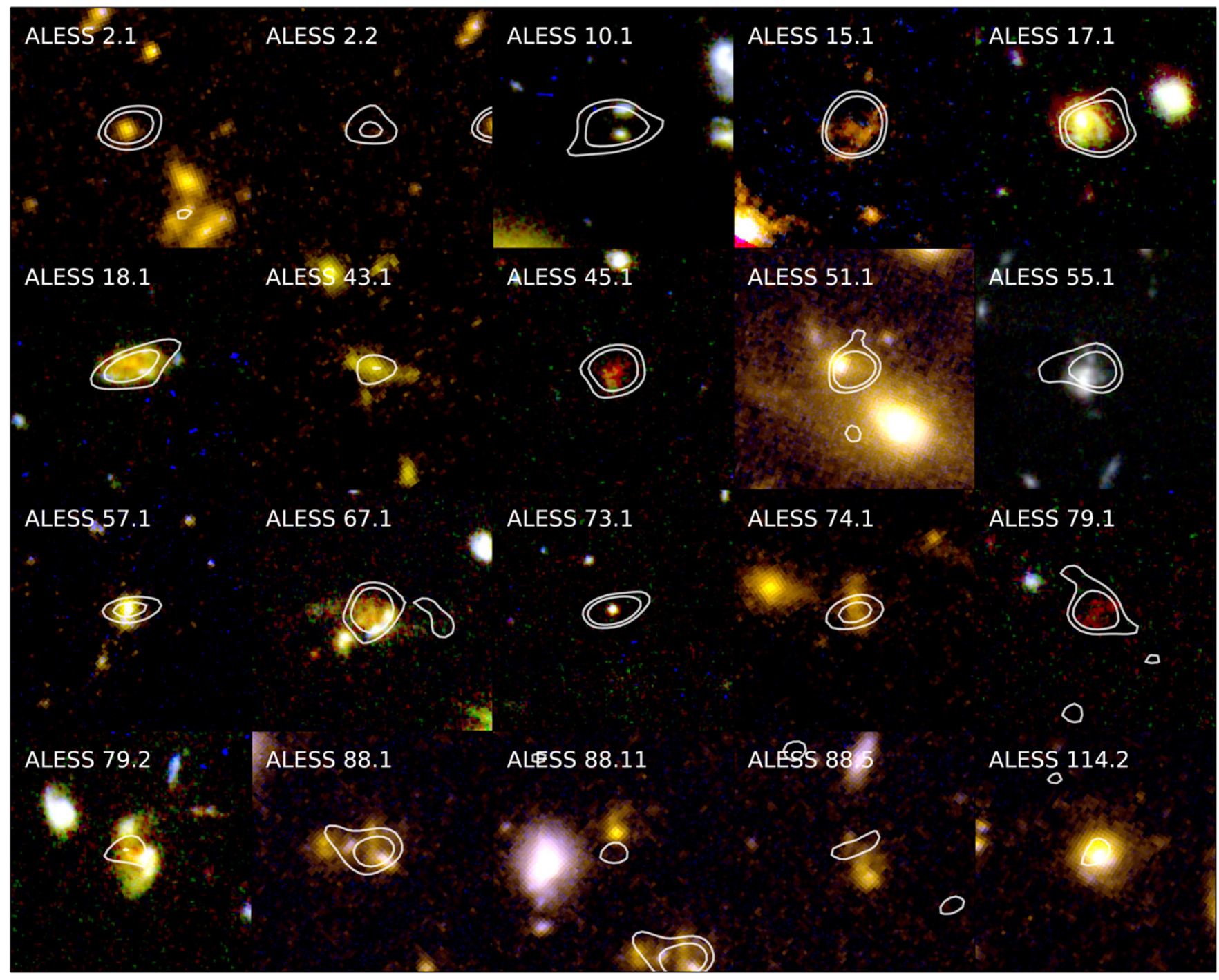

Figure 1. $H S T$ false-color images of 20 out of $25 H_{160}$-detected ALESS SMGs that have imaging in at least two $H S T$ bands, to demonstrate the diversity in morphologies present within the sample. In these maps the red and blue channels are WFC3 $H_{160}$ and ACS $I_{814}$, respectively, except for ALESS 55.1, where the blue is WFC3 $J_{105}$. The green channel is WFC3 $J_{125}$ if available, or if not, we made artificial green maps by interpolating $H_{160}$ and $I_{814}$. These images show that SMGs have a mix of morphology classes, but the majority, $\sim 80 \%$, appear to be irregulars or interacting systems in the rest-frame optical. The contours show the submillimeter emission from our ALMA $870 \mu \mathrm{m}$ maps with levels at $3 \sigma$ and $5 \sigma$. The size of each box is $\sim 70 \times 70 \mathrm{kpc}$ based on the photometric redshifts $\left(\sim 8^{\prime \prime} \times 8^{\prime \prime}\right.$ at $\left.z \sim 2\right)$.

5. Unclassified-sources that are contaminated by nearby bright stars, galaxies, or image artifacts, or are too faint to be classifiable.

Note that the first three classes are not mutually exclusive; one source can be classified as a combination of different classes, such as Disk + Spheroid or Disk + Spheroid + Irregular.

If there are multiple counterparts located within the ALMA synthesized beam of a single SMG, we tagged those sources as either "interaction" or "close companions." Sources that have tidal tails or low-level emission connecting multiple counterparts were tagged as an interaction, while those that do not have apparent interacting features were tagged as close companions. An interaction tag may also represent a source that appears to be in the final coalescent stage of a merger, with asymmetric merger remnants. We show examples for each class along with the two additional tags in Figure 3 (see also Figures 4 and 5 in Kartaltepe et al. 2014). These tags are independent from the main classifications described above. For example, an SMG such as ALESS 49.1 can be classified as Spheroid + Irregular with an interaction tag.
Four of our team members (C.-C.C., I.R.S., J.M.S., C.-J.M.) determined visual classifications of the $H_{160}$-detected SMGs, as well as the comparison sample, and we derived from their classifications the median fraction of all main morphological classes and the bootstrapped errors, which are later used in our analysis.

\subsection{GALFIT}

To quantify the morphology of the SMGs, we fitted a Sérsic profile to the $H_{160}$-band surface brightness of each individual counterpart galaxy, using the most recent version of GALFIT (v3.0.5; Peng et al. 2010). GALFIT is a two-dimensional (2D) fitting algorithm that is designed to fit the surface brightness distribution of a source with various predefined models, such as the Sérsic profile (de Vaucouleurs 1948; Sersic 1968), which is described as

$$
\Sigma(r)=\Sigma_{e} \exp \left[-\kappa\left(\left(\frac{r}{r_{e}}\right)^{1 / n}-1\right)\right],
$$



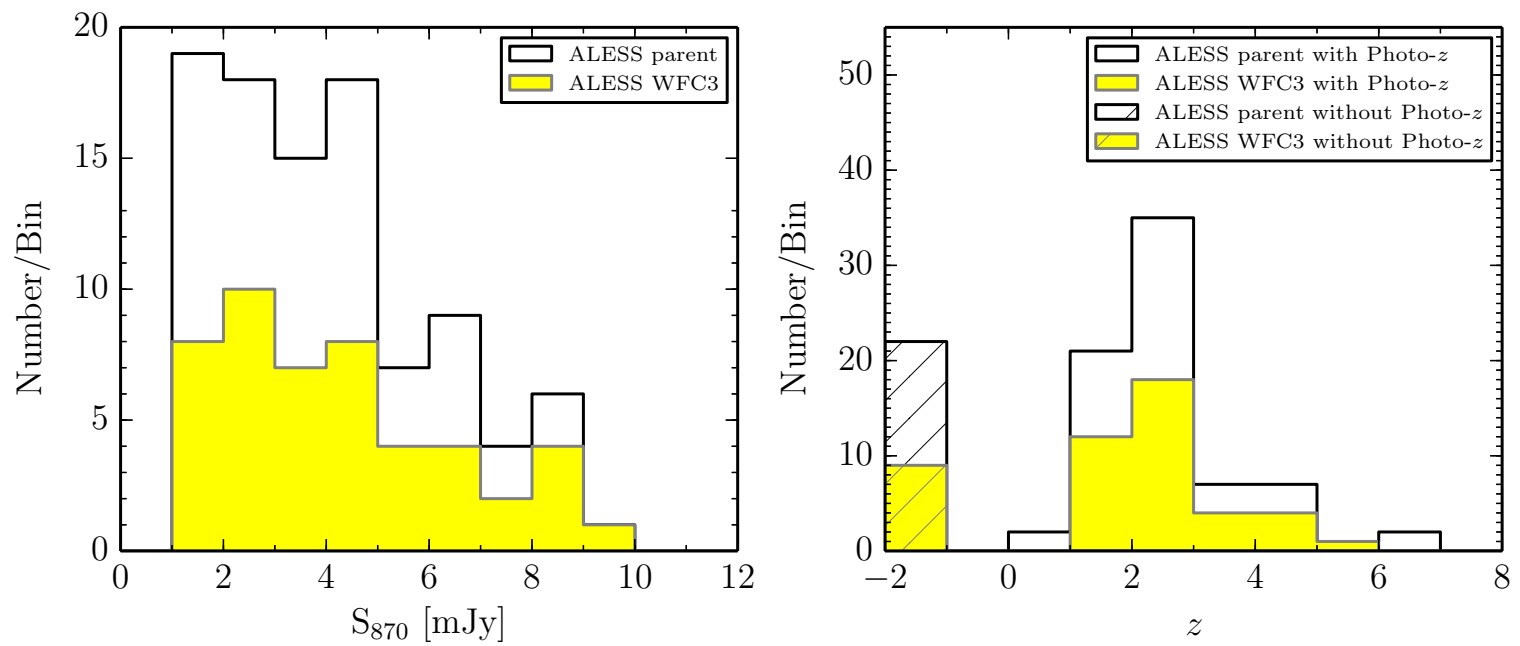

Figure 2. Left: histogram showing the distribution of fluxes from the MAIN catalog of the ALESS survey (Hodge et al. 2013) (black solid line). The yellow region shows the distribution of fluxes for the MAIN ALESS SMGs that were covered by our $H S T$ WFC $3 / H_{160}$-band imaging. Right: photometric redshift distribution for the MAIN ALESS SMGs (black solid line). The median redshift for the 77 MAIN ALESS SMGs with photometric redshift measurements is $z=2.3 \pm 0.1$, with a tail out to $z>5$ (Simpson et al. 2014). The yellow region again shows the redshift distribution for the $H S T$ WFC3/ $H_{160}$-band observed MAIN ALESS SMGs. The hatched region shows those MAIN ALESS SMGs that do not have redshift measurements (manually assigned to $z=-2$ ). The K-S test shows that the $H S T$-observed ALESS SMGs are drawn from the parent ALESS SMG population with a confidence level of $>99.5 \%$. Note that we treated the two pairs ALESS 55.1/55.5 and ALESS 67.1/67.2 as two single sources in this paper (see Subsection 2.1 for details).

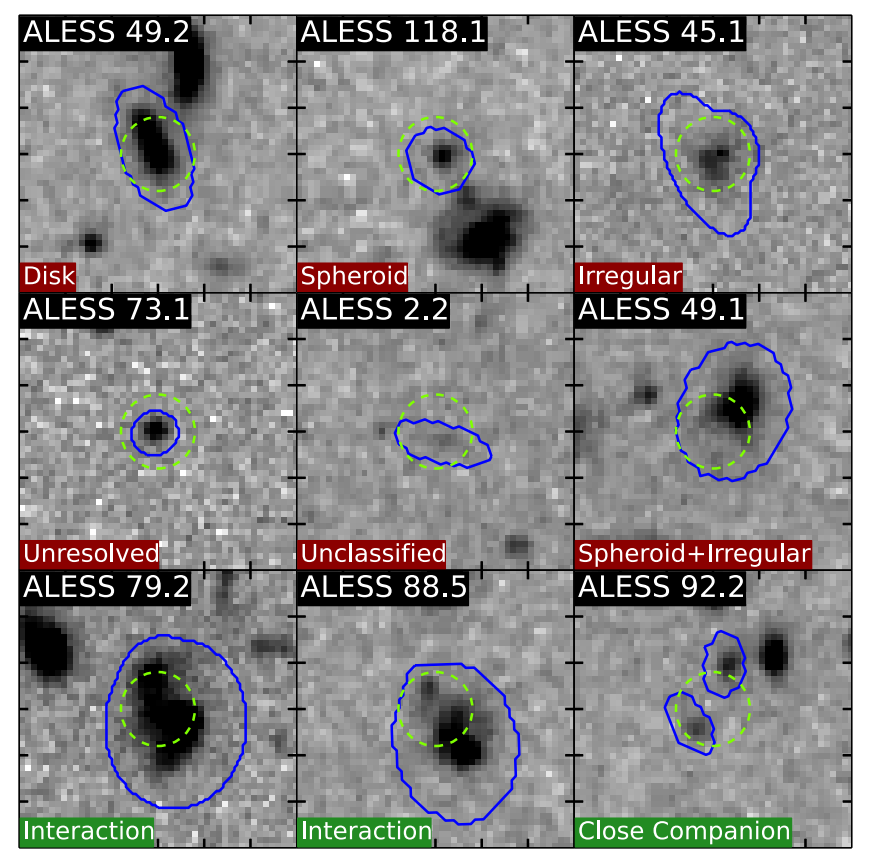

Figure 3. Example thumbnails illustrating the different morphological classifications. Each panel is $6^{\prime \prime} \times 6^{\prime \prime}$, and the tick marks show $1^{\prime \prime}$ increments. Green dashed circles show the typical ALMA beam (1".6 FWHM), and the blue contours enclose the regions where we fit the Sérsic profile (see Subsection 3.2 for detail). The main morphological classes are shown in red, while the two additional classification tags are labeled in green. Images of all $60 \mathrm{H}_{160}$-band observed ALESS SMGs can be found in Figures 10-13 and Tables 1 and 2.

where $\Sigma_{e}$ is the surface brightness at effective radius $r_{e} ; n$ is the Sérsic index indicating the concentration of a light profile, with higher values indicating more concentrated profiles; and $\kappa$ normalizes the fit so that the effective radius $\left(r_{e}\right)$ equals the half-light radius, where half of the total source flux is emitted within $r_{e}$. We report the $r_{e}$ measured along the semimajor axis, which is the direct output of GALFIT.
The key GALFIT inputs for each source are a science image, a PSF map, a "sigma" (or weight) map, a mask outlining pixel regions to be considered in the model fitting, and the first guess for the parameters of the Sérsic profile. Below we describe how we generated these inputs in detail.

The best way to generate a PSF is to median-stack nearby unsaturated and background-subtracted bright stars that are Nyquist sampled (FWHM > 2 pixels; Morishita et al. 2014). This can be achieved in CANDELS and HUDF09, as mosaics were obtained through subpixel dithering, resulting in a finer pixel scale. We therefore generated the PSF images following this procedure for sources covered by these two data sets. However, our newly obtained HST maps have a pixel resolution of $\sim 0{ }^{\prime} .13$. Comparing to the $H_{160}$-band PSF size (FWHM $\sim 0^{\prime}$. 18 ), they are not Nyquist sampled. To circumvent this issue, we first used TINYTIM (Krist et al. 2011), a standard PSF simulation software package for HST, to model the PSF profiles based on the pixel locations of our sources. The PSF profiles were modeled with a pixel resolution three times higher than that of the original HST images (oversampling factor of three). We then fitted stars close to our sources by allowing GALFIT to convolve the model PSFs with Gaussian functions. Finally, we used the best-fit Gaussian functions to convolve the model PSFs to generate the properly centered, effective PSFs that we later used for Sérsic fits.

Input science images were cut into $20^{\prime \prime} \times 20^{\prime \prime}$ thumbnails centered at the ALMA SMG positions. The local background sky and dispersions were measured by fitting Gaussian functions to the pixel distribution of the thumbnails.

For our new WFC3 observations, we used GALFIT to generate the sigma images. In order to do so, GALFIT requires the science images to be calibrated in units of electrons $\left(e^{-}\right)$. We therefore convert the unit of our drizzled images from $e^{-} \mathrm{s}^{-1}$ to $e^{-}$ by multiplying the drizzled images by the exposure time. We generated sigma images for sources covered by CANDELS and HUDF09 by taking the inverse square root of the weight maps. The weight maps of CANDELS/HUDF09 imaging represent pure inverse variance including all background noise terms (sky level for each exposure, read noise, dark current, and flat-field 
structure). However, the final combined mosaics also contain significant amounts of correlated noise, mostly owing to the fact that the PSF is undersampled by the detector pixels. Smaller pixel scale mosaics were created through subpixel dithering, however, at the cost of correlated noise. We therefore manually scaled the CANDELS/HUDF09 sigma images such that the variance of the signal-to-noise $(\mathrm{S} / \mathrm{N})$ distribution was unity, and we derived correction factors of typically $\sim 40 \%-50 \%$. Note that we also used the sigma maps generated by GALFIT instead of the weight maps for CANDELS-observed SMGs using the simulated exposure maps publicly available on the CANDELS website, and we found no significant difference in our results.

The input science thumbnails were masked during fitting to prevent the background sky from dominating the $\chi^{2}$ values. We used SEXTRACTOR to generate the segmentation maps used in masking. Each detected source is described as an elliptical shape in SEXTRACTOR, and a set of parameters, $C_{X X}, C_{X Y}$, and $C_{Y Y}$, are provided in the output catalogs. The parameters are used to parameterize the elliptical shape as $C_{X X}\left(x-x_{0}\right)^{2}+$ $C_{Y Y}\left(y-y_{0}\right)^{2}+C_{X Y}\left(x-x_{0}\right)\left(y-y_{0}\right)=R^{2}$, where $R$ is a scaling parameter, in units of semimajor axis (or semiminor axis), and $x_{0}$ and $y_{0}$ are pixel positions of the source center. The $H_{160^{-}}$ band counterparts of the ALESS SMGs are selected to be any source that is located within the radius of a typical ALMA synthesized beam $\left(00^{\prime \prime} 8\right)$. We generated the masks based on the shape parameters of each counterpart, with $R$ ranging from 3 to 6 depending on the structures of each source. The masks are outlined in blue contours in Figure 3.

The initial values for the Sérsic profile are based on the flux, semimajor axis, semiminor axis, and positional angle in the output catalogs of SEXTRACTOR. In general, for each source we first obtained a well-constrained fit with the initial parameters by varying the mask scaling parameter $R$. We settled with the smallest $R$ that allowed us to obtain a fit. We then used the parameters of this fit as the input for the next iteration. We repeated this process until the output parameters converged. Note that we normally did not place restrictions on the range of any parameters during the fit. However, owing to complicated structures such as tidal tails or very disturbed/faint surface brightness in some systems, mostly involving a multicomponent fit, we fixed the Sérsic index to either 1 or 4 (depending on the preferred index early in the iteration process) on a small number of components. Fixing this index prevents GALFIT from producing unconstrained results with abnormally large/small values that are likely to be unphysical (e.g., a Sérsic index of 20 with an $r_{e}$ of $200 \mathrm{kpc}$ ). As discussed in Section 4.2, fixing the Sérsic index in the model fit for a small number of sources $(\sim 15 \%)$ does not affect our conclusions.

\section{RESULTS}

We summarize the results of our visual classification and profile fitting of the $48 H_{160}$-observed ALESS SMGs in Table 1. In total we detected $38 \mathrm{SMGs},{ }^{16}$ with a detection rate of $79 \% \pm 17 \%$. In addition, $61 \% \pm 16 \%$ (23 out of 38 ) of the $H_{160^{-}}$-detected ALESS SMGs have more than one $H_{160^{-}}$ band component based on Table 1 . In contrast, only $\sim 10 \%$

\footnotetext{
16 Note that ALESS 5.1 is strongly contaminated by a nearby bright galaxy and our SEXTRACTOR settings could not distinguish the two. We therefore measured the $H_{160}$-band flux of ALESS 5.1 using aperture photometry with a 4 pixel $(\sim 0$.'54) diameter aperture and a local sky estimate. We varied the sky annulus to estimate the systematic uncertainties, which were included in the errors, and selected the value that produced the best signal-to-noise ratio $(\mathrm{S} / \mathrm{N} \sim 6)$.
}

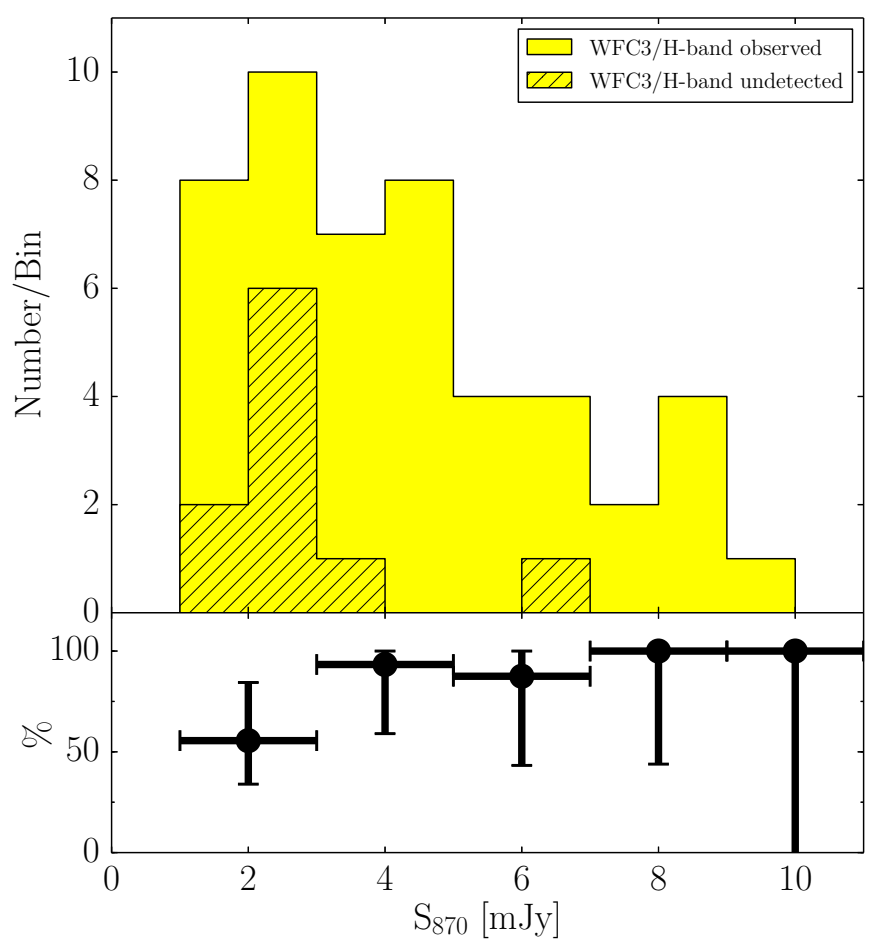

Figure 4. Upper: histogram of the $870 \mu \mathrm{m}$ flux distribution of the $H_{160}$-bandobserved and the $H_{160}$-band-undetected ALESS SMGs. Lower: $H_{160}$-band detection rate of the ALESS SMGs in each flux bin. The errors were estimated through Poisson statistics. Most $(\sim 80 \%)$ of the $H_{160}$-undetected SMGs are submillimeter faint with $S_{870}<3$ mJy.

(5 out of 58) of the comparison sample has multiple $H_{160}$-band components.

In Figure 4 we plot the $870 \mu \mathrm{m}$ flux distribution of the ALESS SMGs that were observed in $H_{160}$ band, as well as those that were undetected in our $H_{160}$-band imaging. As shown in the figure, most of the $H_{160}$ nondetections are for SMGs with $S_{870} \sim 1-3 \mathrm{mJy}$, and the fraction of $H_{160}$-band-undetected ALESS SMGs increases at fainter $870 \mu \mathrm{m}$ fluxes (Figure 4). A similar trend of decreasing detection rate for fainter ALESS SMGs was also seen at the radio/MIR wavelengths (Hodge et al. 2013). In fact, this trend continues to even fainter SMGs with $S_{870}<1 \mathrm{mJy}$ (Chen et al. 2014). These $H_{160}$-undetected SMGs are potentially high-redshift sources that are too faint to be detected in infrared/radio data at the current sensitivities (Dannerbauer et al. 2002; Chen et al. 2014; Simpson et al. 2014).

We plot in Figure 5 the $H_{160}$-band magnitude against $870 \mu \mathrm{m}$ flux of the ALESS SMGs, color-coded by their redshifts. Although the SEXTRACTOR and GALFIT measured flux densities for all the $H_{160}$-band-detected ALESS SMGs (except ALESS 5.1 as a result of strong foreground contamination) are in agreement, for the total $H_{160}$-band flux densities we adopted those measured using SEXTRACTOR since they are direct measurements and are not model dependent. Again, most of the $H_{160}$-band-undetected SMGs have $S_{870}<3 \mathrm{mJy}$, and in fact, the brightest SMG that is not detected in the $H_{160}$-band imaging, ALESS 1.1 (see Figure 5), is located only a few arcseconds away from a bright foreground galaxy, suggesting that it may be gravitationally lensed and its intrinsic flux may be fainter. While there is no obvious correlation between $H_{160}$-band magnitude and $870 \mu \mathrm{m}$ flux, a trend of SMGs with fainter $H_{160}$ magnitudes lying at higher redshifts can be seen. Based on the relation between the rest-frame $H_{160}$-band magnitude and redshift for ALESS SMGs at $z<2.5$, Simpson et al. (2014) crudely estimated the redshifts 


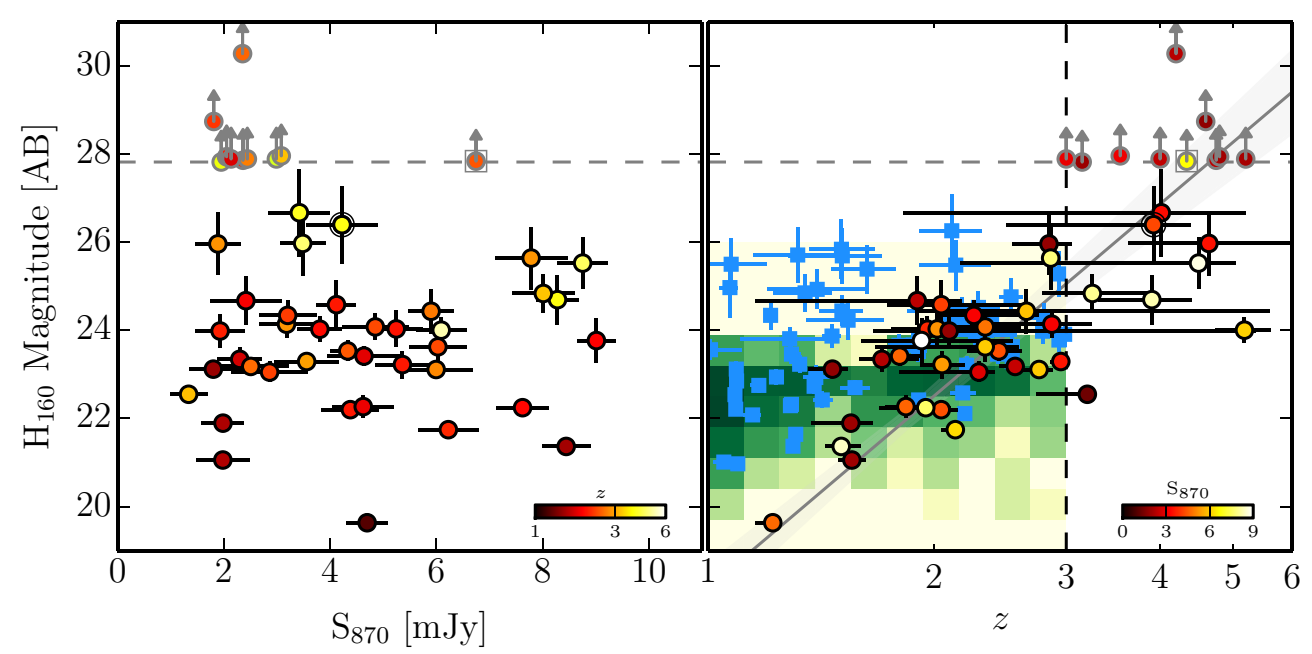

Figure 5. Left: total $H_{160}$-band magnitude vs. $870 \mu \mathrm{m}$ flux for the $48 H_{160}$-band observed ALESS SMGs. The median detection threshold of the $H_{160}$-band imaging is shown as the gray dashed line (27.8), and the nondetections (10) are plotted as gray circles with limits. Each SMG is color-coded based on their redshift. ALESS 1.1, which is potentially lensed by nearby foreground galaxies, is additionally enclosed by a gray square. In addition, ALESS 2.2, enclosed by a black circle, is the only source that is detected in $H_{160}$ band but has an unconstrained photometric redshift, and for this a crude redshift estimate is derived from the relation between the rest-frame $H_{160}$-band magnitude and redshift seen for ALESS SMGs at $z<2.5$ (Simpson et al. 2014). Right: $H_{160}$-band magnitude vs. redshift, with each data point color-coded based on its $870 \mu \mathrm{m}$ flux. While there is no obvious correlation between the $H_{160}$-band magnitude and the $870 \mu \mathrm{m}$ flux, a positive correlation exists between $H_{160}$-band magnitude and redshift, and the best linear regression fit is shown as a gray line with $1 \sigma$ errors in shading. The vertical black dashed line marks the redshift $z=3$ below which our $H_{160}$-band flux measurements are complete. The blue squares are the results of the ALESS comparison sample as described in Section 2.3, and the two-dimensional histogram in green shows the source number density based on the MUSYC catalog in the ECDFS field (Cardamone et al. 2010). The ALESS SMGs are among the brightest $H_{160}$-band sources at $z<2$, possibly suggesting that they are among the most massive systems across this redshift range.

(adopted in this plot) of these $H_{160}$-band-undetected ALESS SMGs and suggested that most of them are likely to be at $z>3$.

We can see this trend more clearly in the right panel of Figure 5, where we plot $H_{160}$-band magnitude against redshift for the ALESS SMGs. Note that ALESS 2.2 (enclosed by a circle in both panels) is the only SMG that is detected in $H_{160}$ band but unconstrained in photometric redshift, and the statistically estimated redshift described above is adopted (for illustrative purposes only). A lack of low-redshift, $H_{160}$-faint SMGs is unlikely to be a selection effect given that our $H_{160}$-band imaging is deep enough to detect them, if they exist. On the other hand, we might miss a few very rare high-redshift $H_{160}$-band bright galaxies that a survey with a larger area coverage than our original LESS survey is capable of detecting. At $z<3$, where our $H_{160}$-band flux measurements are complete (Figure 5), we derive a nonparametric Spearman's rank correlation coefficient of 0.51 with a probability of $99.6 \%$ ( $p$-value of $0.4 \%$ ) that a correlation exists between $H_{160}$-band magnitude and redshift. We conducted linear least-squares fits to our data at $z=1-3$ (where the $H_{160}$-band measurements are complete) and found a best-fit relation of $H=(18.2 \pm 0.6)+(14.5 \pm 2.0) \times \log _{10} z$ with a reduced $\chi^{2}=0.7$. A lack of correlation in the $H_{160}-S_{870}$ diagram implies no or weak correlation between $S_{870}$ and $z$, which confirms the finding of Simpson et al. (2014), however, again with the caveat that the redshifts for the fainter SMGs are incomplete.

The correlation we found for our ALESS SMGs between the $H_{160}$-band magnitude and redshift is in general agreement with the previous SMG studies, showing a similar trend between $K$ band magnitude and redshift (Dannerbauer et al. 2004; Smail et al. 2004; Clements et al. 2004; Chen et al. 2013; Barger et al. 2014). Interestingly, at $z<2$, comparing to either the comparison sample or the field galaxies across the whole ECDFS from the MUSYC catalog, the correlation appears to trace the bright $H_{160}$-band envelop of the field populations, possibly suggesting that SMGs are among the most luminous and hence potentially massive systems at $z<2$. At $z>2$, ALESS SMGs occupy a similar locus in the $H-z$ diagram to the field population. However, the intrinsic $H_{160}$-band flux of $z>2$ SMGs could be much higher given that at these redshifts the $H_{160}$ band traces rest-frame wavelengths blueward of the $V$ band, where the SMGs will suffer more significantly from dust obscuration. Deep and longer-wavelength observations are needed to investigate whether SMGs are also among the most brightest, and thus the most massive, systems, at $z>2$.

Our $H_{160}$-band flux measurements of the ALESS SMGs are complete up to $z=3$ thanks to the deep $H_{160}$-band imaging. To make a clean assessment of our sample of SMGs, we now focus our analysis on the 29 (excluding ALESS 5.1 as a result of strong contamination) $H_{160}$-band detected ALESS SMGs at $z=1-3$ (Figure 5), unless otherwise stated. The results for $z>3$ SMGs are included in Section 4.1 for illustration, and our results are in fact insensitive to the redshift cut. By making this redshift cut, we include most of the SMGs that are bright enough in the $H_{160}$ band for a visual classification, and we found that more than $95 \%$ of the $H_{160}$-detected ALESS SMGs at $z<3$ are classifiable. In addition, at $z<3$ the $H_{160}$-band imaging effectively maps rest-frame optical emission $\left(\lambda_{\text {rest }}>4000 \AA\right)$ and hence provides a more reliable tracer of the stellar light distribution.

\subsection{Visual Classifications}

The visual classification method described in Section 3.1 shows that many of our systems have structural elements that fall in more than one morphological class, reflecting the complexity of their structures (as a result, because the classification bins are not mutually exclusive, the percentages of SMGs classed in different morphological bins in the following do not sum to $100 \%)$.

Our visual classification finds that $61_{-11}^{+12} \%$ of the $H_{160}$-banddetected ALESS SMGs at $z=1-3$ include a component with a 
disk-like morphology (including pure disks and any combination of disk and the other main classes such as disk+spheroid or disk+irregular). We also find that $37_{-8}^{+10} \%$ of the SMGs are either pure spheroids or include a component with spheroidal morphology, and that $58_{-12}^{+13} \%$ are pure irregulars or include a component that appears irregular. Moreover, $52_{-10}^{+11} \%$ of the SMGs appear to have features indicative of interaction. Combining the latter two classes, we find that the percentage of SMGs with disturbed morphologies, meaning they either are irregular or have an interaction tag, is $82 \% \pm 9 \%$. In contrast, we found that only $48 \% \pm 6 \%$ of the comparison far-infrared-faint field sample has disturbed morphologies, suggesting that these are relatively dynamically calmer or more isolated systems.

We compare our classifications with those from Kartaltepe et al. (2012), who presented similar visual classifications of a galaxy sample lying within the central region of the GOODS-S field and selected at 100 and $160 \mu \mathrm{m}$ using the PACS instrument (Poglitsch et al. 2010) on board the Herschel Space Observatory (Herschel), as part of the GOODS-Herschel program (Elbaz et al. 2011). The ULIRGs presented by Kartaltepe et al. (2012) have a mean $L_{\mathrm{IR}}$ of $2 \times 10^{12} L_{\odot}$ and a mean redshift of $z=2.2$; compared to the $z \sim 2$ ALESS SMGs, $L_{\mathrm{IR}} \sim 3.0 \times 10^{12} L_{\odot}$ and $z=2.1$, the ULIRG sample from Kartaltepe et al. (2012) has a slightly lower mean $L_{\mathrm{IR}}$ but a similar mean redshift. These ULIRGs are also expected to be hotter in terms of dust temperature than the ALESS SMGs and potentially more AGNdominated, since they are selected at rest-frame $\sim 30-50 \mu \mathrm{m}$. In addition, Kartaltepe et al. (2012) present a non-Herschelselected comparison LIRG sample with redshift and $H_{160}$-band magnitude distributions matched to their ULIRG sample but lower infrared luminosity $\left(L_{\mathrm{IR}}<3.2 \times 10^{11} L_{\odot}\right)$, which is also plotted in Figure 6.

We show this comparison in Figure 6, where we see that for all morphological classes, the distributions of $z \sim 2$ hot ULIRGs from Kartaltepe et al. 2012 are consistent with the $z \sim 2$ ALESS SMGs, while the results for the ALESS SMGs differ significantly in the categories related to disturbed morphology from those of LIRGs in Kartaltepe et al. (2012), as well as compared to our comparison low-luminosity field sample. Hence, although at $z=1-3$ submillimeter-selected SMGs preferentially pinpoint the galaxies with lower dust temperature than the hot far-infrared-selected ULIRGs presented by Kartaltepe et al. (2012), it appears that the morphological mix seen in the ALESS SMGs is similar to that seen in comparably luminous PACS-selected ULIRGs at similar redshifts.

We next investigate the sensitivity of our classifications to the effects of redshift, in particular the morphological $K$ correction: the variation in source morphology arising from color variations within galaxies, due to dust or age effects, coupled with the sampling of different rest-frame wavelengths at different redshifts (e.g., Kartaltepe et al. 2014). While we focus our analysis on $z<3$ SMGs, where the $H_{160}$-band fluxes more closely trace the bulk of the stellar emission, we can test whether the results of our visual classifications vary with redshift, in particular in the $z>3$ regime, where the $H_{160}$-band imaging samples the rest-frame UV emission. It is possible that the rest-frame UV morphologies of $z>3$ SMGs will appear more irregular than that seen in the rest-frame optical/nearinfrared sampling of the $z<3$ SMGs since the rest-frame $\mathrm{UV}$ is more sensitive to clumpy star-forming regions and dust obscuration.

In the bottom panel of Figure 6 we investigate the fraction of SMGs in each morphological class in high- and low-redshift

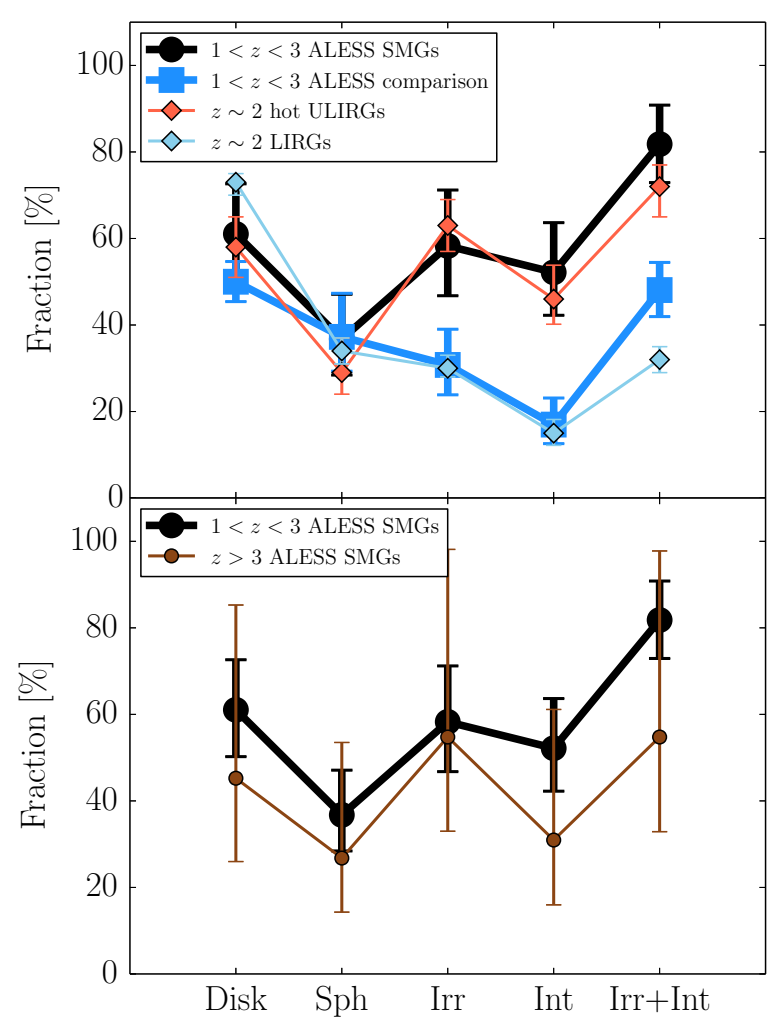

Figure 6. Upper: fraction of the $H_{160}$-band-detected ALESS SMGs at $z=1-3$, $z \sim 2$ hot ULIRGs selected at $100 / 160 \mu \mathrm{m}$, and $z \sim 2$ LIRGs in each of the morphology classes (Disk, Disk; Sph, Spheroid; Irr, Irregular; Int, Interaction). The results for the $z \sim 2$ hot ULIRGs and LIRGs are taken from Kartaltepe et al. (2012). The Irr+Int class represents a combined class of irregular and interaction. Note that because the morphological classes are not mutually exclusive, the percentages do not sum to $100 \%$. Lower: same as the upper panel, but we now compare SMGs at $z<3$ and those at $z>3$. The fraction of $z>3$ SMGs with disturbed morphologies is slightly lower than (but statistically comparable to) that of $z<3$ SMGs, confirming that our results of a high irregular fraction for SMGs are robust and not obviously affected by morphological $K$-correction.

subsamples. Although suffering from small number statistics in the $z>3$ subsample, the fraction of $z>3$ SMGs with disturbed morphologies is slightly lower than (but statistically consistent with) that of lower-redshift SMGs. This is mostly due to the $H_{160}-z$ relation in Figure 5, which shows that higherredshift SMGs have lower $H_{160}$-band fluxes. This trend results in many $z>3$ SMGs appearing as a single, faint source in the $H_{160}$-band imaging, and they are unlikely to be classified as a clumpy irregular. The lower fraction of irregulars at $z>3$ is thus likely to be due to the depth of the available imaging, and deeper images are needed to investigate the true morphology of $z>3$ SMGs. On the other hand, our results of a high irregular fraction at $z<3$ are robust and not affected by morphological $K$-correction.

It is also worth noting that the presence of AGNs in the sample does not seem to affect our conclusions. There are six ALESS SMGs that are identified as AGNs through X-ray detections (these are noted in Table 1; Wang et al. 2013), with five of these at $z=1-3$. However, we do not observe a significant difference in the visual classes between AGN SMGs and non-AGN SMGs.

\subsection{Sérsic Index and Effective Radius}

In Figure 7 we plot the Sérsic index $(n)$ and the effective radius $\left(r_{e}\right)$ for every component of the $z=1-3$ ALESS SMGs detected in our $H_{160}$-band imaging as a function of infrared luminosity, redshift, and $H_{160}$-band magnitude. 


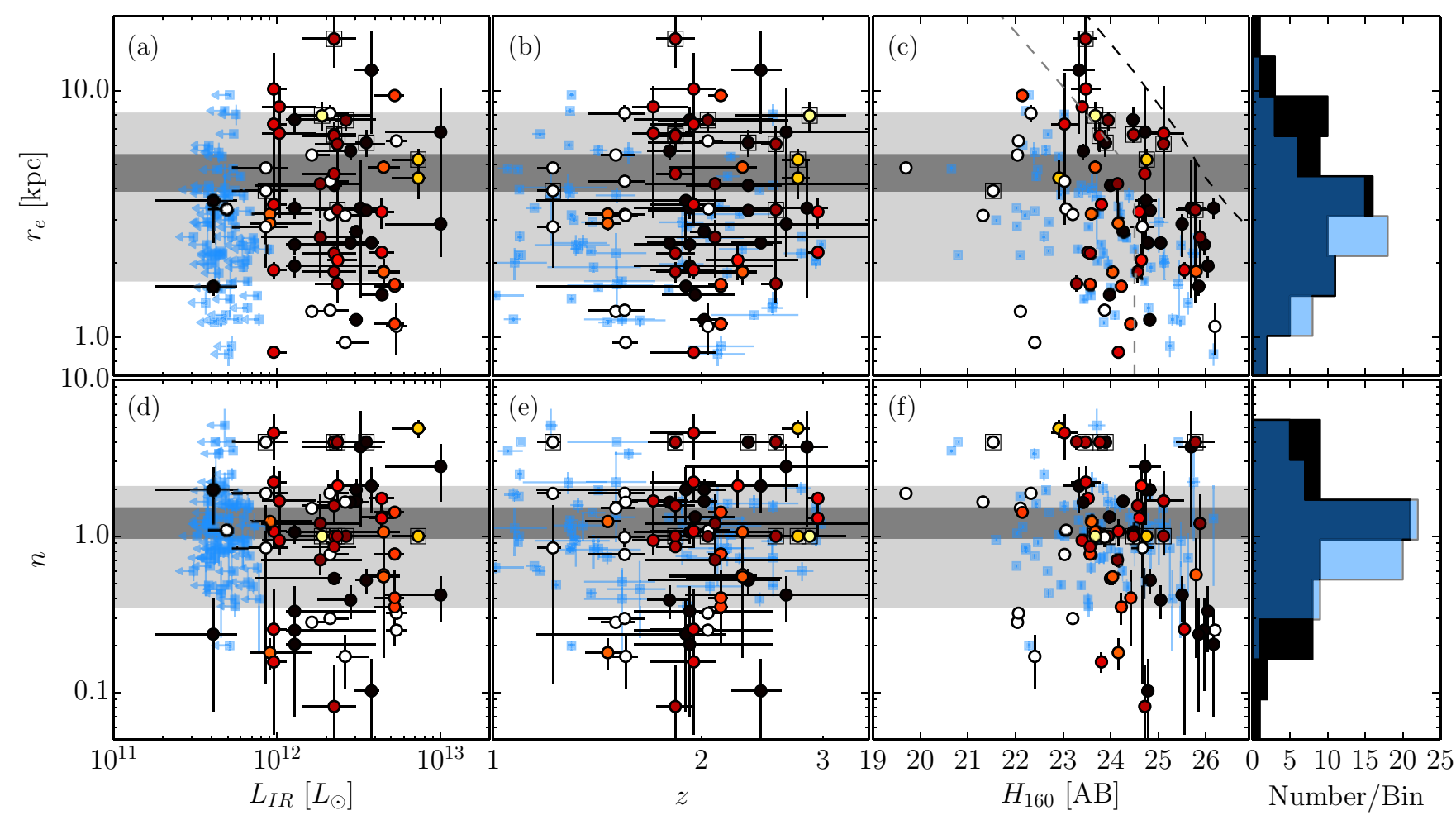

Figure 7. Panels (a)-(f) show the distribution of effective radius $\left(r_{e}\right)$ and Sérsic index $(n)$ as a function of total luminosity $\left(L_{\mathrm{IR}}\right.$; derived by Swinbank et al. 2014), redshift ( $z$; Simpson et al. 2014), and the $H_{160}$-band AB magnitude for $z=1-3$ ALESS SMGs. The last panel of each row shows the histogram of either $n$ or $r_{e}$, in which black and blue represents ALESS SMGs and the comparison sample, respectively. The ALESS SMGs are color-coded based on their $\chi^{2}$ values from the GALFIT model fit (see Table 1), and those enclosed by an extra black square are those with a fixed Sérsic index. The results of the comparison sample are shown in blue. The gray (black) dashed curves in panel (c) represent the maximum $r_{e}$ detectable based on Equation (7) at the sensitivity of the previous NICMOS studies (our new $H_{160}$-band imaging). The dark gray horizontal bands are the median values of $H_{160}$-band components with $H_{160} \leqslant 24$ for ALESS SMGs (unbiased $r_{e}$ at $<15 \mathrm{kpc}$; also see the text in Section 4.2), with the width showing the bootstrapped errors. The light gray horizontal bands represent the $1 \sigma$ intrinsic scatter. There is no detectable variation in $n$ or $r_{e}$ with $L_{\mathrm{IR}}, z$, and $H_{160}$ for the ALESS SMGs from our the $H_{160}$-band imaging. While the sources in the comparison sample are consistent with the ALESS SMGs in terms of $n\left(1.0 \pm 0.1\right.$ vs. $1.2 \pm 0.3$ respectively), on average they have a smaller $r_{e}$ than the SMGs $\left(2.5 \pm 0.3 \mathrm{kpc}\right.$ vs. $\left.4.4_{-0.5}^{+1.1} \mathrm{kpc}\right)$.

Overall there are no obvious trends in any combination of parameters for the ALESS SMGs, regardless of the goodness of the fit $\left(\chi_{v}^{2}\right)$. However, systems with higher $\chi_{v}^{2}$ tend to be at lower redshifts and have brighter $H_{160}$-band fluxes, suggesting that low surface brightness features such as tidal tails or clumpy structures, which are only revealed in these closer and brighter systems, drive the higher values of $\chi_{v}^{2}$. Indeed, by adding noises into the thumbnails for these high $\chi_{v}^{2}$ systems and rerunning the GALFIT analysis, we found consistent best-fit solutions but with lower $\chi_{v}^{2}$ values. We therefore concluded that these bright, low-redshift but high- $\chi_{v}^{2}$ systems are not intrinsically different in $n$ and $r_{e}$ from those at higher redshift with fainter $H_{160}$-band fluxes.

On the other hand, an absence of sources with large $r_{e}$ and faint $H_{160}$ components can be seen in Figure 7 ; in the following we argue that this is due to the sensitivity of the $H_{160}$-band imaging.

We can rewrite the Sérsic profile (Equation (1)) in a simpler form as

$$
\Sigma(r)=\Sigma_{o} \exp \left[-\kappa\left(\frac{r}{r_{e}}\right)^{1 / n}\right]
$$

where $\Sigma_{o}=\Sigma_{e} e^{\kappa}$ is the central surface brightness, and the total flux would be

$F=\int_{0}^{2 \pi} d \phi \int_{0}^{\infty} r \Sigma(r) d r=2 \pi \Sigma_{o} \int_{0}^{\infty} r \exp \left[-\left(\frac{r}{\alpha}\right)^{1 / n}\right] d r$ where $\alpha=r_{e} / \kappa^{n}$. We then make a substitution of $t=(r / \alpha)^{(1 / n)}$, and thus $r=t^{n} \alpha$ and $d r=\alpha n t^{n-1} d t$. Finally, Equation (3) becomes

$$
F=2 \pi \Sigma_{o} \alpha^{2} n \int_{0}^{\infty} t^{2 n-1} \exp (-t) d t
$$

and the integral is actually a Gamma function $(\Gamma(y)=$ $\left.\int_{0}^{\infty} x^{y-1} \exp (-x) d x\right)$. Thus, Equation (4) becomes

$$
F=2 \pi \Sigma_{o} \alpha^{2} n \Gamma(2 n)
$$

and

$$
\Sigma_{o}=\frac{F}{2 \pi \alpha^{2} n \Gamma(2 n)} .
$$

We then substitute this back into Equation (2), and we now have

$$
r_{e}^{2} \exp \left[\kappa\left(\frac{r}{r_{e}}\right)^{1 / n}\right]=\frac{\kappa^{2 n} F}{2 \pi n \Gamma(2 n) \Sigma(r)} .
$$

Equation (7) means that, taking our deepest surface brightness sensitivity $\left(\Sigma\left(r_{\mathrm{det}}\right)\right)$ and the total number of pixels required for the SEXTRACTOR detection threshold, we can determine the radius $\left(r_{\text {det }}\right)$ corresponding to any total flux $F$ and hence solve for the maximum detectable $r_{e}$ given any $n$ ( $\kappa$ is a function of $n$ ). In practice, for a given $F$ ( $H_{160}$ in our case), we solved for the maximum $r_{e}$ using Equation (7) for a range of Sérsic profiles 
( $n=4,2,1,0.5,0.1$, and the corresponding $\kappa=7.67,3.67,1.68$, $0.70,0.02$, respectively) and converted $r_{e}$ to kpc by adopting the mean redshift $z=2.1$. We plot the results in Figure 7 . This selection line bounds the parameter space of our observations and demonstrates that for a given detection limit there is a size bias toward smaller $r_{e}$ when detecting components close to the detection limit. Thus, one needs to be cautious when comparing typical sizes between two samples if they were observed to different depths.

The median effective radius (or half-light radius, $r_{e}$ ) of all $H_{160}$-band components in Table 1 is $3.3 \pm 0.2 \mathrm{kpc}$ (all the errors quoted on median values in this paper were obtained through a bootstrapping method). However, if we only take components that have $H_{160} \leqslant 24$, which should be complete for measured sizes up to $\sim 15 \mathrm{kpc}$, we obtained a median $r_{e}$ of $4.4_{-0.5}^{+1.1} \mathrm{kpc}$ (dark gray horizontal band in the top panels of Figure 7), with an intrinsic scatter of the $H_{160}$-band component sizes of $1.7-8.1 \mathrm{kpc}$. Applying an $H_{160}$-band cut at $H_{160} \leqslant 24$ provides a fairer estimate of the rest-frame optical sizes for ALESS SMGs, assuming that the $r_{e}$ distribution at $H_{160}>24 \mathrm{mag}$ is not drastically different from that at $H_{160} \leqslant 24 \mathrm{mag}$. Note that our measurements of the median $r_{e}$ are not sensitive to the $H_{160}$ cut between 22 and 24 mag.

A NICMOS study by Swinbank et al. (2010) of the $H_{160^{-}}$ band morphologies of a sample of 25 radio-identified SMGs determined a median $r_{e}$ of $2.8 \pm 0.4 \mathrm{kpc}$, consistent with our results based on all $H_{160}$-band components but somewhat lower than our unbiased measurements with an $H_{160}$ cut. ${ }^{17}$ However, considering the shallower sensitivity of NICMOS imaging $\left(\mu_{H} \sim 24.5 \mathrm{mag} \operatorname{arcsec}^{-2}, 1 \sigma\right)$, we might expect a bias toward low $r_{e}$. Indeed, we show the NICMOS $r_{e}$ sensitivity as the dashed gray curve in panel (c) of Figure 7. The median $r_{e}$ of our $H_{160}$-band components that meet the NICMOS sensitivity is $3.2 \pm 0.5 \mathrm{kpc}$, consistent with the results of Swinbank et al. (2010), which we conclude were biased down somewhat by the surface brightness limit of their study.

Recently, Targett et al. (2013) used the CANDELS $H_{160}$-band imaging to study the morphology of a sample of a mixture of millimeter and submillimeter sources uncovered by AzTEC at $1.1 \mathrm{~mm}$ and LABOCA at $870 \mu \mathrm{m}$ (the latter taken from the same LESS survey used as the basis of ALESS). They found that their sample of 24 candidate counterparts to the submillimeter sources has a mean $r_{e}$ of $4.3 \pm 0.5 \mathrm{kpc}$, consistent with our results. With a comparable $H_{160}$-band depth to our study, it is perhaps not surprising that both groups found a consistent median $r_{e}$. However, without high-resolution follow-up observations with interferometers, their results are very likely to suffer from contamination by misidentifications ( $\sim 20 \%$; Hodge et al. 2013), for example, the ALMA observations show that their proposed counterpart to LESS J033217 is not the SMG. In addition, the selection function for this sample is complicated by the use of a heterogeneous submillimeter and millimeter sample, where the different depths, resolutions, and selection wavelengths will bias the sample in different ways in redshift and/or dust temperature.

The surface brightness sensitivity of our WFC3 imaging tends to bias the mean $r_{e}$ of the ALESS SMGs toward artificially smaller values at faint magnitudes. However, as shown in panel

\footnotetext{
17 We should note that Swinbank et al. (2010) defines the half-light radius $\left(r_{h}\right)$ as the radius at which the flux is one-half of that within the Petrosian radius. Based on Graham et al. (2005), a profile with $n \leqslant 1 r_{h}$ equals $r_{e}$, while $r_{h}$ is only $\sim 70 \%$ of $r_{e}$ for $n=4$. As most SMGs have low $n$, we do not expect $r_{h}$ to be significantly different from $r_{e}$. Indeed, we measured $r_{h}$ on our SMGs with $H_{160} \leqslant 24$ mag following the procedure of Swinbank et al. (2010) and find a median $r_{h}$ of $4.3 \mathrm{kpc}$.
}

(c) of Figure 7 , the $H_{160}$-band imaging is actually sufficiently deep to reveal a trend between $r_{e}$ and $H_{160}$ for the field sample, in which $d\left(\log \left(r_{e}\right)\right) / d\left(H_{160}\right) \sim-0.2$. Given that there is no correlation between $n$ and $H_{160}$ for the comparison sample (panel (f) in Figure 7), based on Equation (5) the trend is consistent with the scenario that the central surface brightness $\left(\Sigma_{0}\right)$ of the comparison field sample remains constant on average across the $H_{160}$ range and the apparent size of the source then determines its integrated brightness. Perhaps more interestingly, we can use the deeper WFC3 data to test the influence of the NICMOS sensitivity limits on the comparison of the field and SMG samples from Swinbank et al. (2010). We find that while the median $r_{e}$ of the whole comparison field sample is $2.5 \pm 0.2 \mathrm{kpc}$, the median $r_{e}$ is $3.1 \pm 0.3 \mathrm{kpc}$ for those sources that meet the NICMOS sensitivity criteria in Swinbank et al. (2010). Hence, if we had to rely on shallower NICMOS imaging, we would have concluded that the ALESS SMGs and the low-luminosity field comparison sample are indistinguishable in $r_{e}$ (this is consistent with the findings in Swinbank et al. 2010). However, employing the full depth of our WFC3 imaging, we can begin to separate these two populations in terms of their typical sizes and see that the SMGs are larger on average than the general field population at these redshifts.

Turning now to the distribution of Sérsic index $(n)$ in Figure 7, we can see that these are highly peaked at low $n$ for both the ALESS SMGs and the comparison sample, with $\sim 80 \%$ of the sources having $n<2$. Indeed, for the ALESS SMGs the median $n$ for those $H_{160}$-band components with $H_{160} \leqslant 24$ mag is $1.2 \pm 0.3$, with a $1 \sigma$ dispersion of $0.4-2.1$. The median $n$ is not sensitive to the $H_{160}$ cut (the median $n$ is $1.0 \pm 0.2$ for all the $H_{160}$-band components), but we make the cut at $H_{160} \leqslant 24 \mathrm{mag}$ just to be consistent with the measurement of the median $r_{e}$. The median $n$ for the sources in the comparison field sample is $1.0 \pm 0.1$. Since there is no apparent bias in the derived Sérsic index as a function of image depth (indeed, we find solutions for a wide range of $n$ in Equation (7) at $H_{160}<27 \mathrm{mag}$ ), it is not surprising that the median $n$ from Swinbank et al. (2010) and Targett et al. (2013), $1.4 \pm 0.8$ and 1.0, respectively, are consistent with our results.

In this analysis we have assumed that every $H_{160}$-band component that overlaps the ALMA synthesized beam is a counterpart of the ALESS SMG. While in most cases this is likely to be true, we do not have redshift measurements to confirm our assumptions, in particular for a few ambiguous cases (e.g., ALESS 10.1 or ALESS 92.2), potentially casting doubts on the robustness of our identifications. We test whether a few ambiguous cases would affect our results by just measuring the most likely component of each ALESS SMG (labeled $\mathrm{H} 1$ in Table 1) ${ }^{18}$ We found the median $r_{e}$ and $n$ unchanged, with $r_{e}=4.1_{-0.8}^{+1.6} \mathrm{kpc}$ and $n=1.2_{-0.3}^{+0.2}$, respectively. However, we found the intrinsic scatter for $r_{e}$ to be slightly smaller, $2.7-7.7 \mathrm{kpc}$, which is likely to be the lower limit to the true scatter as our test did not take into account some secondary components, which are likely to be the counterparts of the ALESS SMGs (i.e., ALESS 49.2, ALESS 57.1, ALESS 79.2, ALESS 87.1). Similarly, the scatter based on all the $H_{160}$-band components as presented earlier is likely to be the upper limit

\footnotetext{
18 In all cases but ALESS 114.2, these are the closest components to the ALMA positions. The $H_{160}$-band counterpart of ALESS 114.2 can be modeled with two components (as can be seen in Table 1, as well as in Figure 10): H1 represents the main component accounting for the bulk of the $H_{160}$-band flux; $\mathrm{H} 2$ models the lower-level emission surrounding $\mathrm{H} 1$. Although $\mathrm{H} 1$ has a slightly larger offset to the ALMA position, H1 is likely to be the representative $H_{160}$-band component for ALESS 114.2.
} 


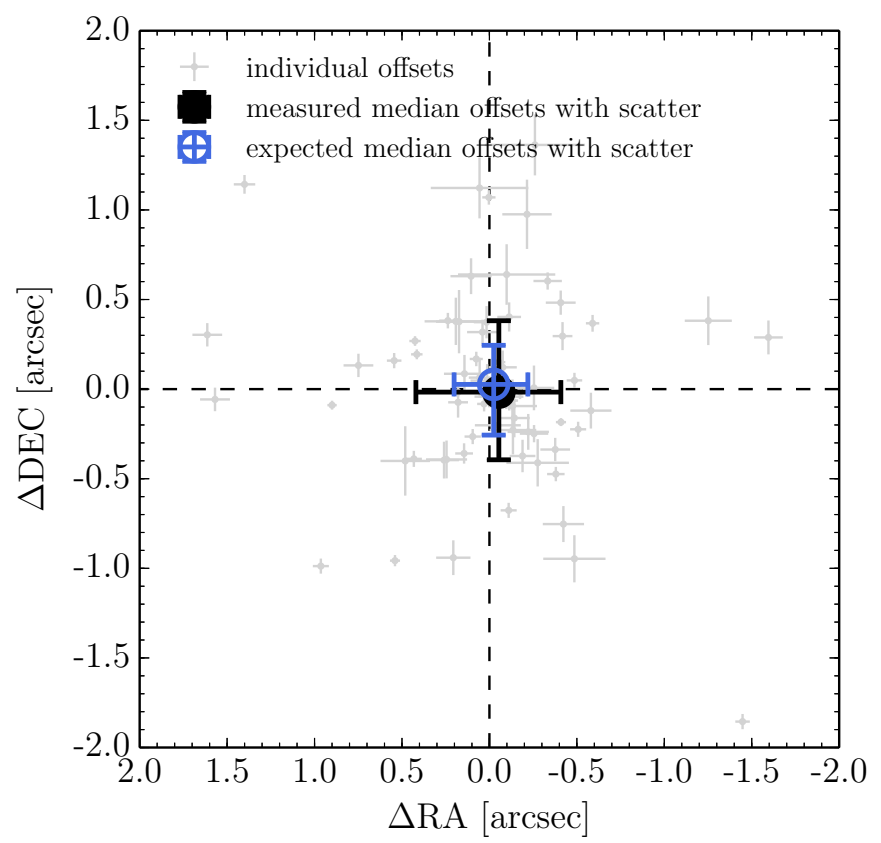

Figure 8. Offsets in R.A. and decl. between the $H_{160}$-band component and the corresponding ALMA $870 \mu \mathrm{m}$ emissions ( $H_{160}-$ ALMA; as quoted in Table 1 ). The individual component is shown in gray, with errors estimated based on the uncertainties from both GALFIT and the ALMA observations. The measured (expected) median offsets are shown in black (blue) circles, with the symbol size corresponding to about two times the error $\left(-0^{\prime \prime} .05 \pm 0.05\right.$ in R.A. and $0.02 \pm 0.06$ in decl.). The intrinsic scatters are shown in black error bars, which on average are $00^{\prime \prime} 4 \pm 0.05$. The significantly larger measured positional scatter compared to that expected suggests that most of the dusty star-forming regions are not located close to the center of the stellar distribution, as seen in the rest-frame optical.

as a few may not be the correct counterparts. We adopted the upper limit of the $r_{e}$ scatter to maintain a consistent approach throughout this paper.

\subsection{Positional Offsets}

With each $H_{160}$-band component of our ALESS SMGs reliably modeled using GALFIT with good positional constraints, as well as the subarcsecond positional accuracy provided by the ALMA imaging, we can investigate the positional relation between the dusty star-forming regions traced by ALMA and the stellar components traced by our $H_{160}$-band imaging.

In Figure 8 we plot the positional offsets in R.A. and decl. between all our $H_{160}$-band components at $z=1-3$ in Table 1 and their corresponding ALMA $870 \mu \mathrm{m}$ positions (defined as $H_{160}-$ ALMA). In addition to the GALFIT errors, for each $H_{160^{-}}$ band component we folded in the positional errors of the original ALMA observations derived by Hodge et al. (2013). We measured a median $\Delta$ R.A. of -0 '.05 \pm 0 '.05 and a median $\Delta$ decl. of $-0.02 \pm 0$. . 06 . We also measured an average intrinsic scatter of $0.40 \pm 0.05$.

As we included in this analysis any $H_{160}$-band component that intersects the ALMA synthesized beam (1".6 FWHM) as potentially part of the SMG, it is possible that some fraction of the components are not associated. Hence, if we restricted our analysis to just those components whose centroids fall within the ALMA beam, we obtained an average intrinsic scatter of $0.34 \pm 0 . .03$ and median offsets of $-0 . .07 \pm 0.05$ in R.A. and $-0.03 \pm 0.06$ in decl.
The systematic offsets from both definitions of the counterparts are not significant, confirming our initial alignment of the astrometric frames described in Section 2.1. As stated in Section 2, the intrinsic scatter between the IRAC $3.6 \mu \mathrm{m}$ sources and their calibrated $H_{160}$-band counterparts is $00^{\prime \prime} 15-0.17$. We expect the scatter between ALMA and IRAC to be comparable to this, meaning that if the stellar components are well aligned with the dusty star-forming regions, we derive that the expected scatter between ALMA and IRAC is $\sqrt{2} \times 00^{\prime \prime} 17=00^{\prime} \cdot 24$, adopting the higher scatter to be conservative. The measured scatter for the ALESS SMGs is $00^{\prime} 4 \pm 0.05$, significantly larger than the expected value, $0^{\prime \prime} 24$. This is still the case if we only consider the scatter obtained from the $H_{160}$-band components located within the typical ALMA beam. In fact, only 34\% (44\%) of the data points (those within the synthesized ALMA beam) are consistent with no offset.

A significantly larger scatter in positional offsets between the $H_{160}$-band components and the corresponding ALMA $870 \mu \mathrm{m}$ peaks suggests that the majority of the dusty star-forming regions are not located close to the center of the stellar distribution seen in the rest-frame optical. This could simply suggest that the dusty star-forming regions are so obscured that the rest-frame optical imaging does not reflect the true stellar morphology. We tested this scenario by comparing the measurements between lower $(z=1-2)$ and higher $(z=2-3)$ redshift subsamples. If obscuration is truly a significant factor and the dusty star-forming regions are indeed located close to the center of the stellar distribution, we would expect the scatter for the low-redshift subsample to be smaller, as the $H_{160^{-}}$ band imaging probes their rest-frame 5000-8000 $\AA$ and is less obscured by dust. However, we found no statistical difference in the positional scatter between the low-redshift and high-redshift subsample. We therefore conclude that it is more likely that the offset between the $H_{160}$-band components and the corresponding ALMA $870 \mu \mathrm{m}$ peaks reflects real misalignment between the dusty star-forming regions and the locations of the majority of the stellar masses within the SMGs.

\section{DISCUSSION}

\subsection{Massive Disks or Mergers?}

Recently, a few studies have analyzed the near-infrared morphologies of SMGs in order to shed light on the stellar distribution of these high-redshift, dusty star-forming sources (Swinbank et al. 2010; Targett et al. 2013; Wiklind et al. 2014). Various quantitative analyses have been conducted, including the CAS method, the Gini/M20 parameters, and the Sérsic indices, and all the studies have claimed that SMGs are massive disks (Targett et al. 2013) and no more likely to appear as major mergers in the rest-frame optical than those more typical starforming galaxies selected in rest-frame UV (Swinbank et al. 2010). It has also been claimed that SMGs that are selected at $870 \mu \mathrm{m}$ represent a more isolated, heterogeneous population (Wiklind et al. 2014) in contrast to $100 / 160 \mu \mathrm{m}$ selected sources at similar redshifts (Kartaltepe et al. 2012).

In this study we have discovered that although our $\mathrm{H}_{160^{-}}$ band-detected ALESS SMGs appear disk-like in quantitative analysis (median $n$ of $1.2 \pm 0.3)$, most $(\sim 80 \%)$ are in fact visually classified as either irregulars or interacting systems. A lack of visual classification on a statistical sample seems to be the one reason that previous studies mistakenly concluded that most SMGs are disks. After all, the nonparametric tools such as CAS and Gini/M20 have limited power to differentiate 
irregulars/interacting systems from disks (Swinbank et al. 2010; Wiklind et al. 2014).

Now, the question becomes whether sources appearing as irregulars or interacting systems are clumpy rotating disks or mergers. Recently, many efforts have been made to shed light on the triggering mechanism of star formation through studies of gas dynamics. Kinematic studies using integral field unit (IFU) spectroscopic observations of ionized atomic lines ( $\mathrm{H} \alpha$ or [O III]) from $z \sim 1-3$ star-forming galaxies have found that some sources that appear as irregulars in the optical/near-infrared are rotationally dominated (e.g., Genzel et al. 2011), although signs of rotationally supported dynamics do not preclude mergers as the merger signatures could be lost due to limited spatial resolution of high-redshift sources (e.g., Gonçalves et al. 2010). However, most of these studies were focused on rest-frame UVselected star-forming galaxies that have lower SFRs than SMGs. Recent, IFU studies of small samples of SMGs, including one of our sources ALESS 67.1, have instead found either a lack of evidence for global ordered rotation or that most SMGs are classified as mergers based on a kinemetic analysis of the velocity and dispersion field asymmetry (Alaghband-Zadeh et al. 2012; Menéndez-Delmestre et al. 2013). These results are consistent with an earlier IFU study on a sample of six SMGs by Swinbank et al. (2006), which shows that these sources have distinct dynamical subcomponents, suggesting a merging/ interacting nature of these systems. Equally, a dynamical study on ALESS 73.1, using high-resolution (0'.5) interferometric observations with ALMA of the [C II] $157.7 \mu \mathrm{m}$ fine-structure line, has found that the gas reservoir traced by this emission has kinematics that are consistent with a rotating disk (De Breuck et al. 2014), and similar gas disks have been claimed in some other high-redshift ULIRGs (e.g., Swinbank et al. 2011; Hodge et al. 2012). Again, this does not preclude a merger origin as a gas disk is expected to re-form during the final coalescence of a gas-rich merger (e.g., Robertson et al. 2006; Hopkins et al. 2009).

In any case, the mixed results from studies of gas kinematics highlight the complications involved in explaining the dynamical history of SMGs based solely on the observations of molecular or ionized gas. In the following we study the triggering mechanisms of SMGs through the evolution of Sérsic index and effective radius based on our results on the collisionless stellar components of the ALESS SMGs.

\subsubsection{Evolution of Size and Sérsic Index}

Owing to their SFRs, bright SMGs are expected to be shortlived, with a lifetime of $\sim 100 \mathrm{Myr}$ (e.g., Chapman et al. 2005), which is similar to the estimated lifetimes based on SMG clustering (e.g., Hickox et al. 2012). Indeed, the median SFR of our ALESS SMGs is $\sim 500 M_{\odot} \mathrm{yr}^{-1}\left(3 \times 10^{12} L_{\odot}\right)$, and for a typical SMG gas mass of $\sim 5 \times 10^{10} M_{\odot}$ (e.g., Bothwell et al. 2013), the time for ALESS SMGs to exhaust their gas reservoir is of order $100 \mathrm{Myr}$. If the quenching of star formation indeed happens shortly after the SMG phase, then the likely descendants of our $z \sim 2$ ALESS SMGs would be $z \sim 2$ quiescent galaxies. Such connections between $z=2-3$ SMGs and $z \sim 2$ quiescent galaxies have been made by other studies using similar arguments on SFRs and stellar mass growth (e.g., Wardlow et al. 2011; Fu et al. 2013; Toft et al. 2014). Unfortunately, while this is a conceptually tidy proposal, there are potential problems with the details of how the transition is made, in particular from the point of view of transforming the stellar distributions. With a well-defined and reliably identified sample of SMGs at $z \sim 2$,

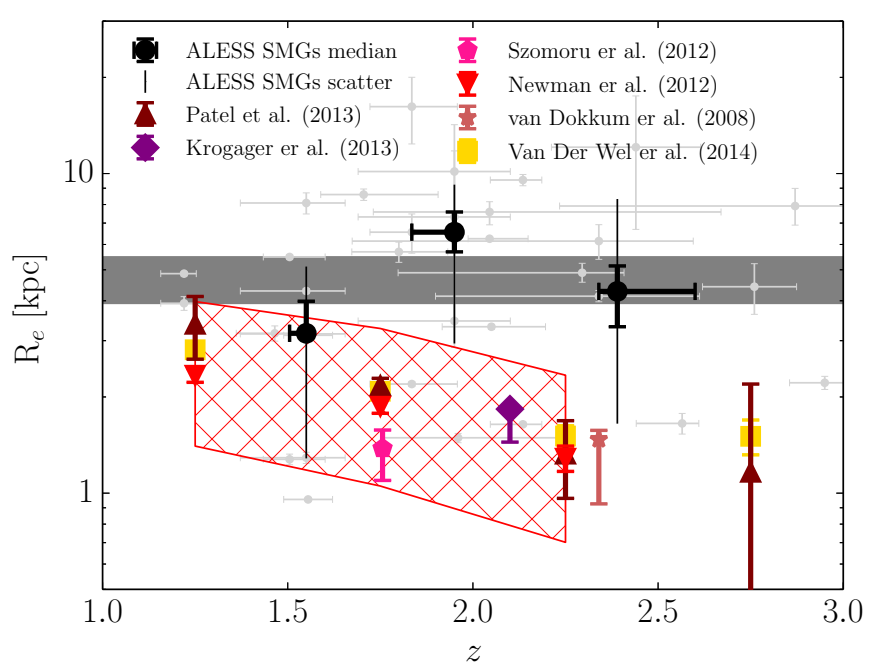

Figure 9. Plot of redshift vs. effective radius $\left(r_{e}\right)$ for different galaxy populations. The results from the individual $H_{160}$-band component of the ALESS SMGs with $H_{160}<24$ are plotted in light gray, with the three equal-sized bins shown in black points and the median size of $4.4 \pm 0.2 \mathrm{kpc}$ in dark gray. The intrinsic scatter in each bin is plotted as vertical black lines. Various measurements from the literature for the sizes of $z \sim 2$ quiescent galaxies are shown, with the typical scatter illustrated by the red grids from Newman et al. 2012. The SMGs are typically 2-3 times larger than $z \sim 2$ quiescent galaxies at a similar epoch.

we can test this proposal and investigate different quenching mechanisms.

In Figure 9 we show the distribution of effective radius $\left(r_{e}\right)$ versus redshift for the $H_{160}$-band components of the ALESS SMGs at $z=1-3$ with $H_{160} \leqslant 24$ mag. We also show the median in three equal number bins (11 SMGs in each) with redshift. As we discussed in Section 4.2, there is no apparent size evolution for the ALESS SMGs. The median size for the ALESS SMGs at $z=1-3\left(r_{e}=4.4_{-0.5}^{+1.1} \mathrm{kpc}\right)$ is shown as the dark horizontal band in Figure 9, and we also plot the size measurements of $z \sim 2$ quiescent galaxies made using the NIC2 camera (van Dokkum et al. 2008), and with the WFC3 in CANDELS imaging in COSMOS (Krogager et al. 2013), GOODS-S (Szomoru et al. 2012), and the UDS field (Newman et al. 2012; Patel et al. 2013). We also include the latest size measurements on apparently passive galaxies at $z<3$ based on the combined CANDELS and 3D-HST surveys (van der Wel et al. 2014). All sizes were converted to the $r_{e}$ measured along the major axis. The conclusions remain unchanged if all $r_{e}$ were instead converted to the commonly used circularized effective radius, $r_{e, \text { circ }}=r_{e} \sqrt{b / a}$, where $b / a$ is the projected axis ratio.

The size difference between SMGs and quiescent galaxies is immediately apparent from Figure 9, especially at $z>2$, where ALESS SMGs are significantly larger than the quiescent galaxies. A large range in size is generally reported for $z=2-3$ quiescent galaxies (e.g., Szomoru et al. 2012; Newman et al. 2012; van der Wel et al. 2014), with a typical scatter of about $0.2-0.25$ dex for mass-selected samples with $\geqslant 5 \times$ $10^{10} M_{\odot}$ (the completeness limit for CANDELS imaging at $z<3$ ). A few $z=2-3$ quiescent galaxies are found to be located within the size range of SMGs, making them candidate descendants of SMGs. However, considering the comoving density of $M_{\star}>5 \times 10^{10} M_{\odot}$ quiescent galaxies at $z \sim 2$, $n_{c} \sim 10^{-4} \mathrm{Mpc}^{-3}$ (Newman et al. 2012; Patel et al. 2013), and the fraction of them with $r_{e}>1.7 \mathrm{kpc}$ (the lower limit of the ALESS SMGs), 10\%-40\% (Trujillo et al. 2007; Newman et al. 2012; Szomoru et al. 2012; van der Wel et al. 2014), the 

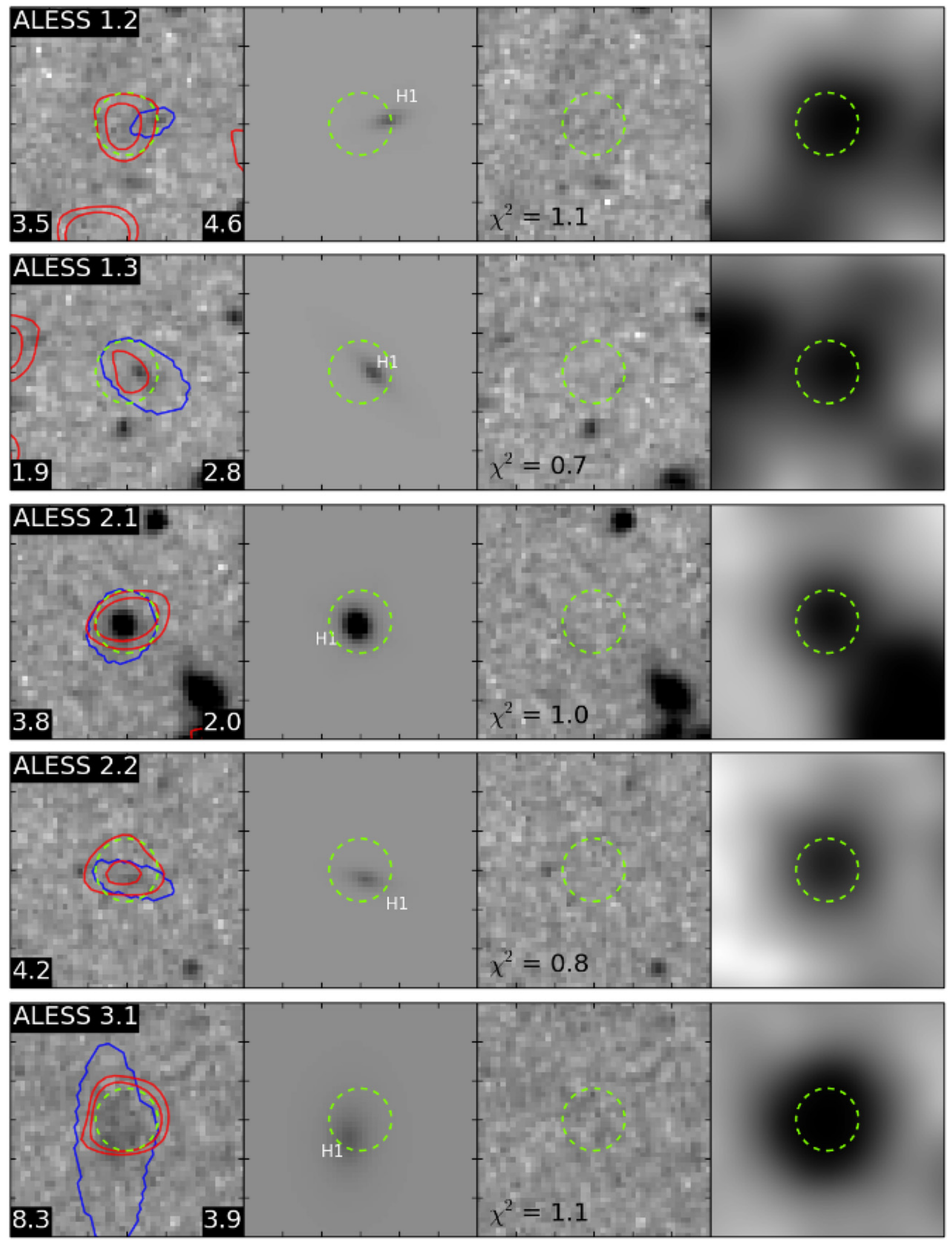

Figure 10. Postage stamps of $H$-band imaging on each MAIN ALESS SMGs. For each source, the panels show (left to right) the original $H$-band imaging, GALFIT Sérsic models within the masked area (blue contours), residuals after subtracting the Sérsic models with the reduced $\chi^{2}$ noted, and the IRAC $3.6 \mu \mathrm{m}$. The size of each box is $6^{\prime \prime} \times 6^{\prime \prime}$, with the green circles representing the typical ALMA synthesized beam of 1".6 FWHM. The ALMA contour at $3 \sigma$ and $5 \sigma$ is shown with red contours in the left panel for each source. The black numbers at the bottom left are $S_{870}$, and those at the bottom right, if any, are redshifts. 

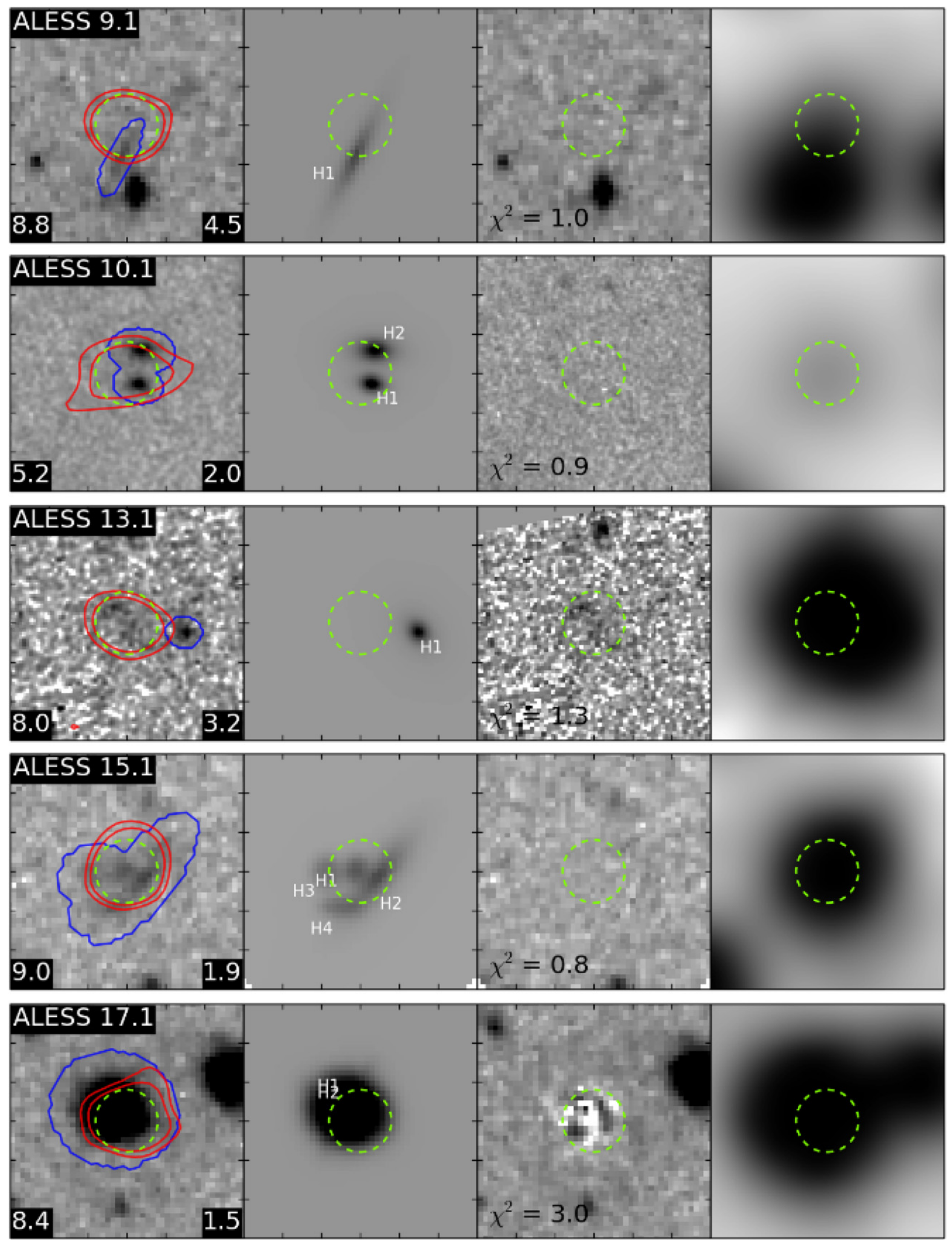

Figure 10. (Continued) 

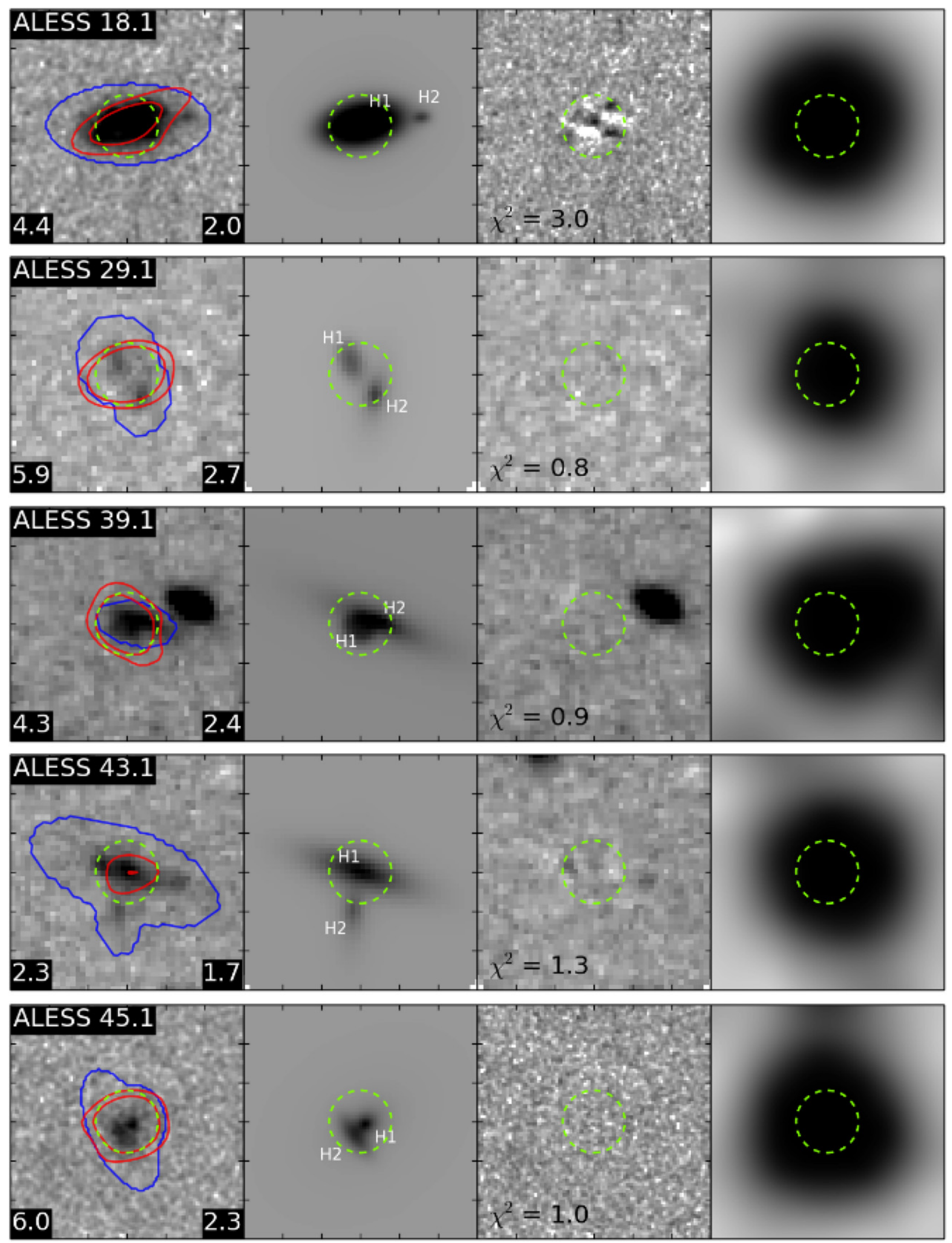

Figure 10. (Continued) 

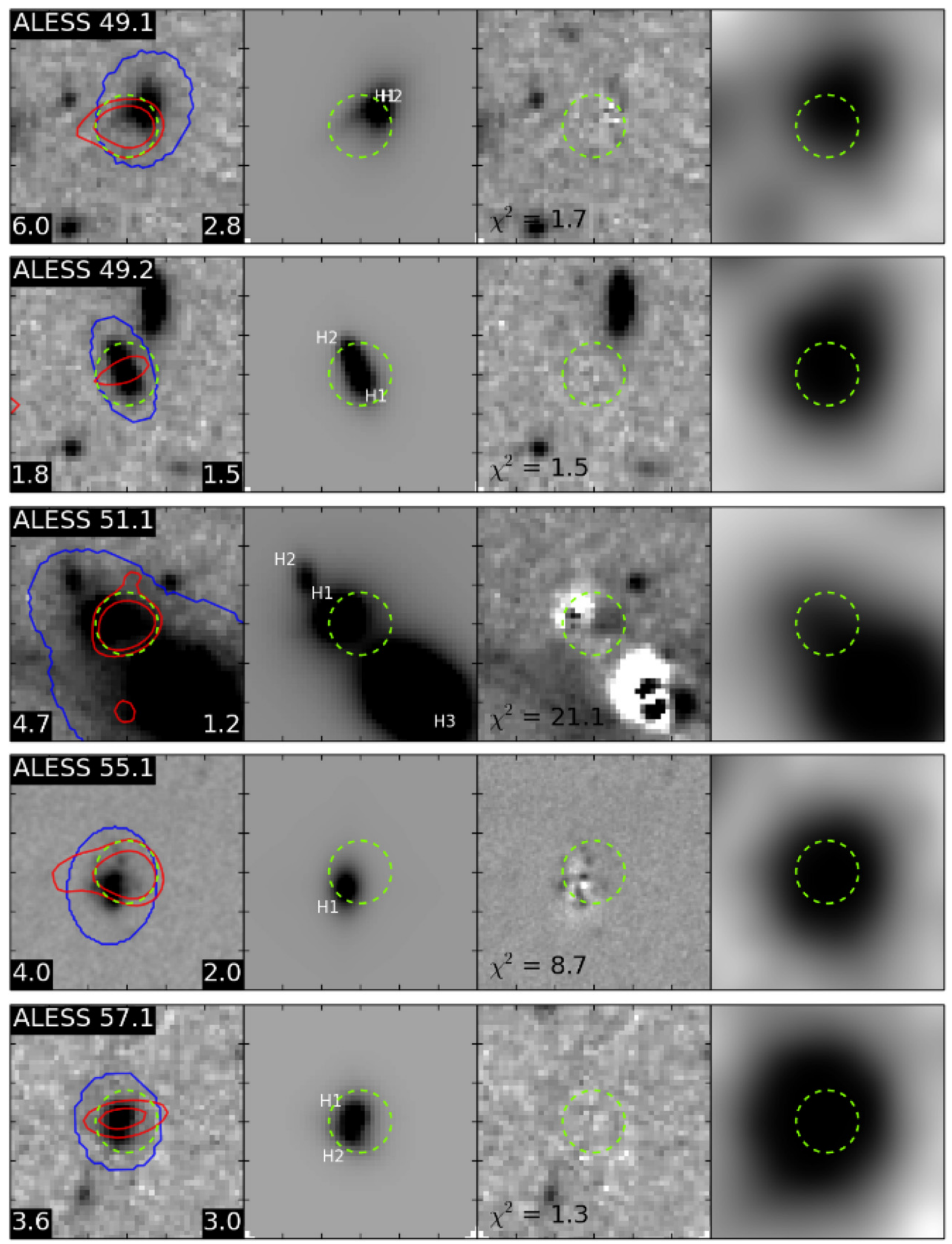

Figure 10. (Continued) 

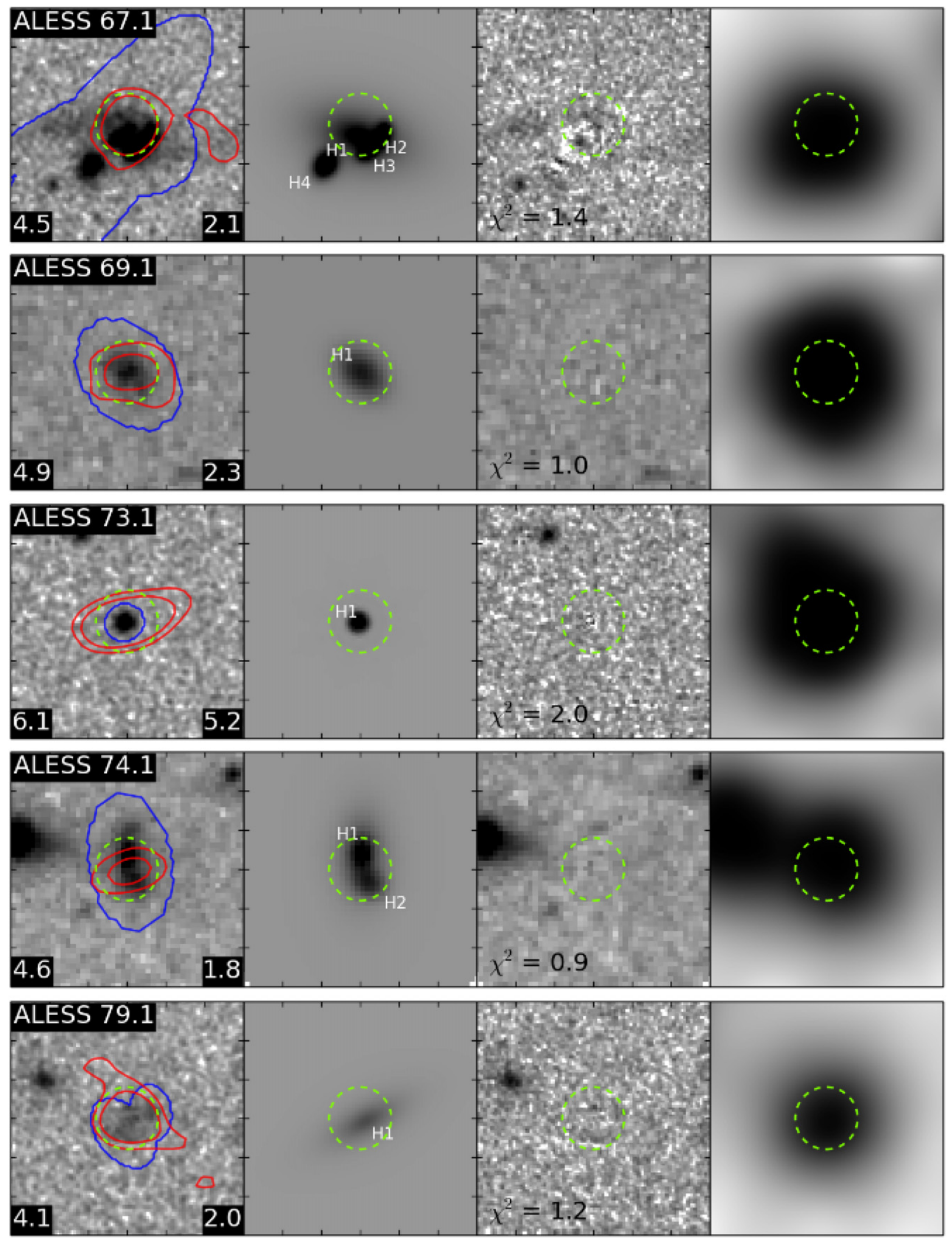

Figure 10. (Continued) 

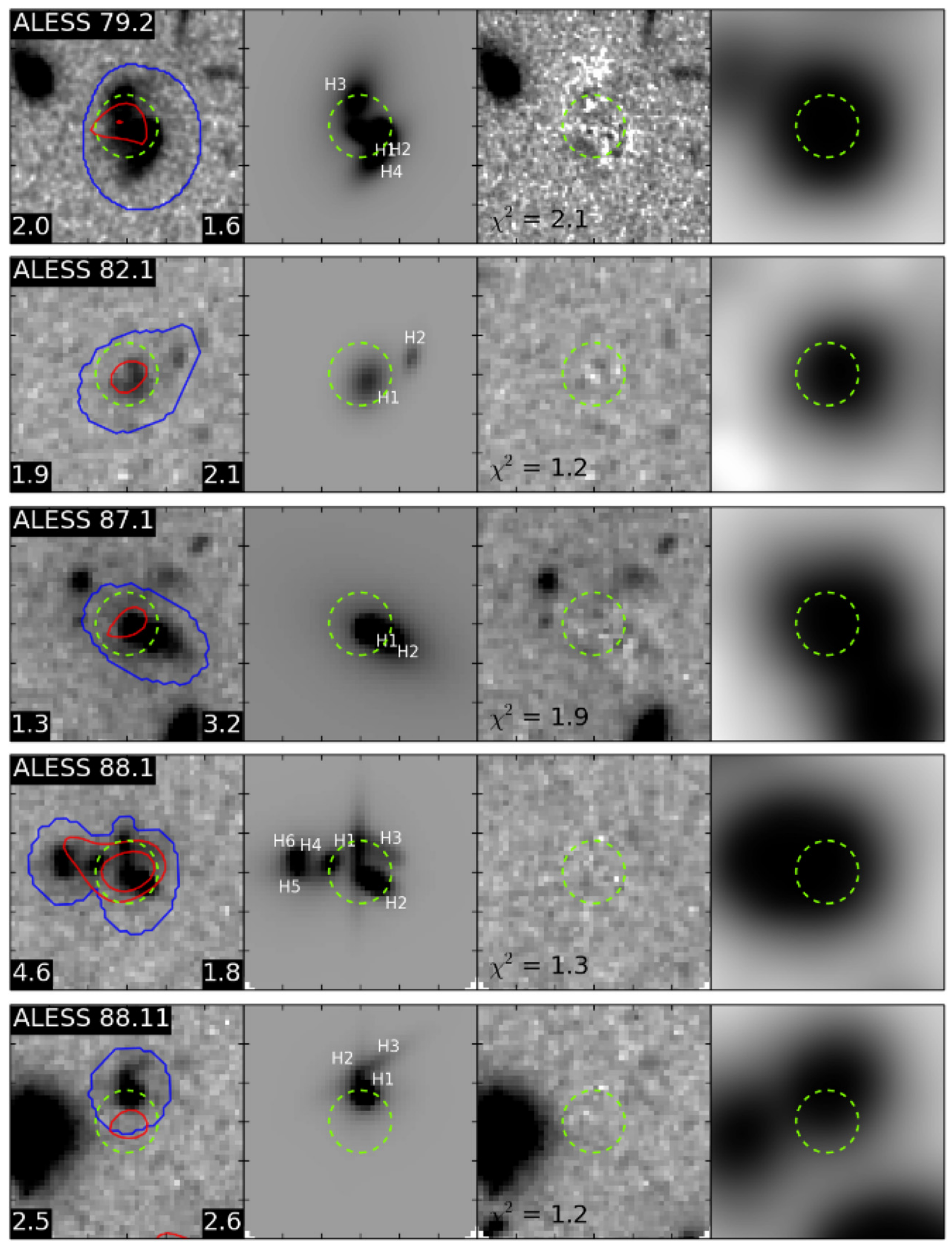

Figure 10. (Continued) 

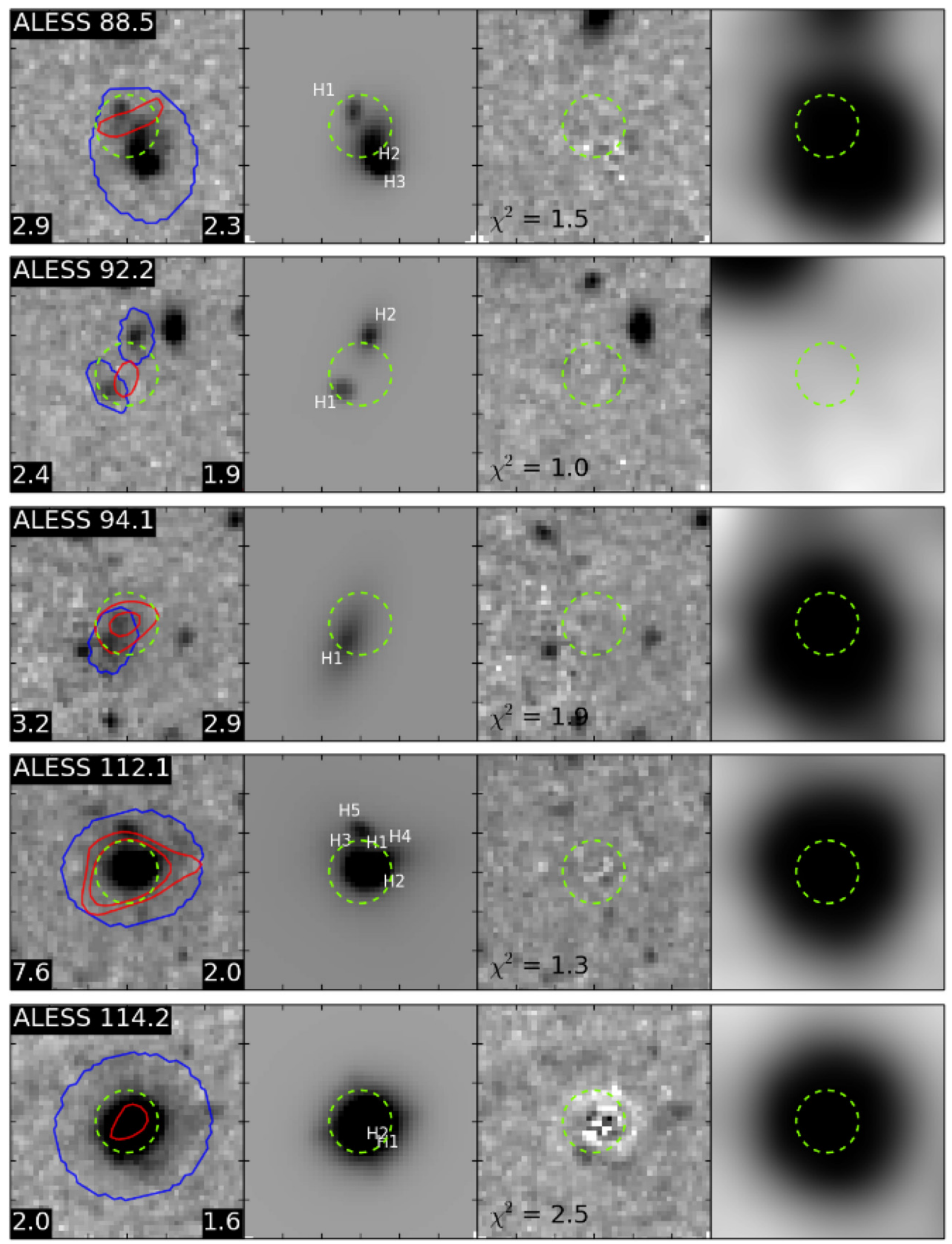

Figure 10. (Continued) 

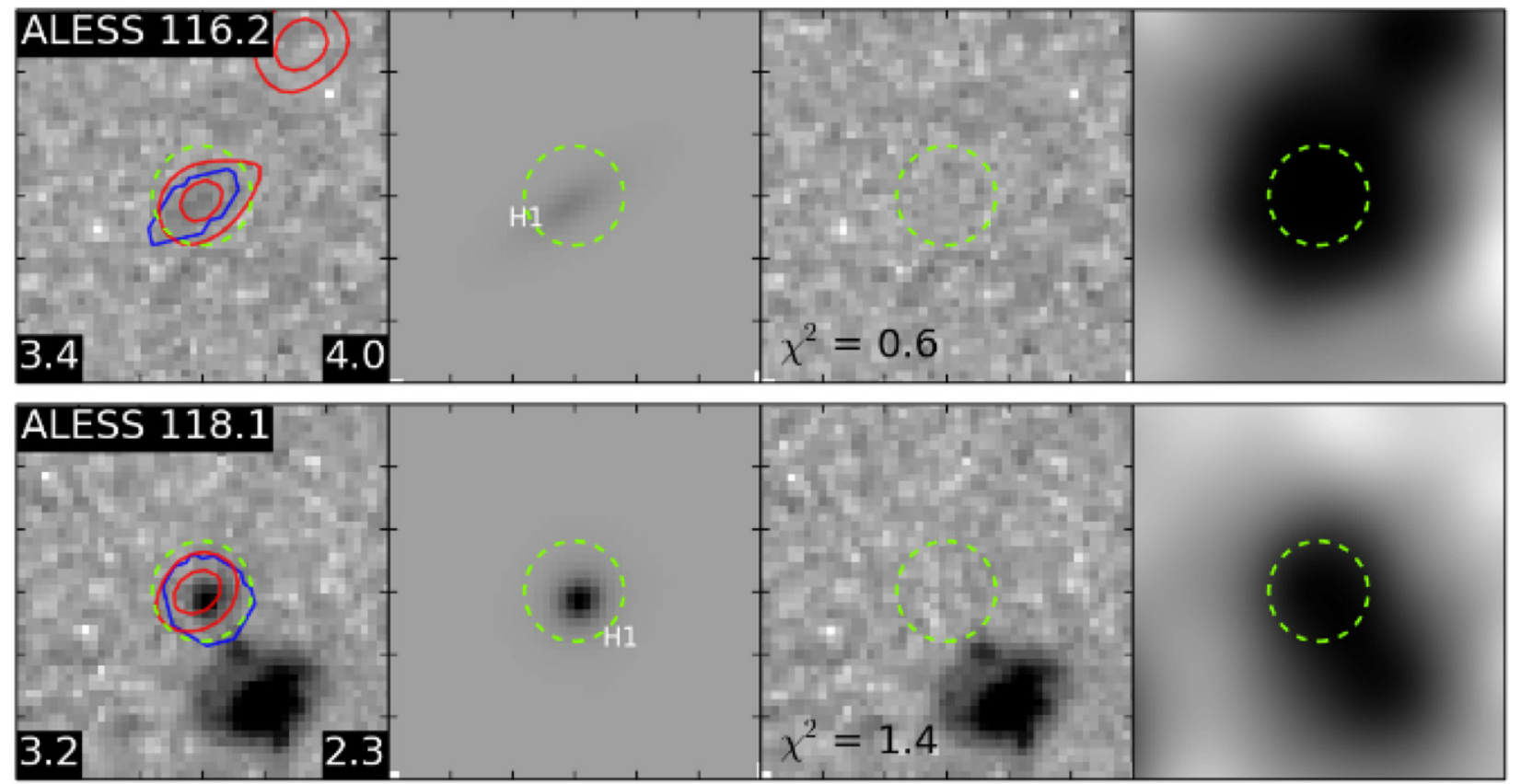

Figure 10. (Continued)

comoving density of $z \sim 2$ quiescent galaxies with $r_{e}>1.7 \mathrm{kpc}$ is only $\sim(1-4) \times 10^{-5} \mathrm{Mpc}^{-3}$. Given the duty cycle, $\sim 10 \mathrm{Gyr}^{-1}$, and the comoving number density, $\sim 10^{-5} \mathrm{Mpc}^{-3}$, of the ALESS SMGs at $z=2-3$ (Simpson et al. 2014), these large $z \sim 2$ quiescent galaxies would only comprise $\sim 10 \%-40 \%$ of the SMG descendants at $z \sim 2$, if they are indeed on the same evolutionary track. This means that if they are to become typical $z \sim 2$ quiescent galaxies, the majority of $z>2$ SMGs have to go through a transformational phase that significantly reduces their stellar half-light radii, as well as increasing their Sérsic indices ( $n \sim 1$ to $\sim 4$ ), before being quenched. Note that the smaller scatter in the sizes, $\sigma_{r e}=1.7 \mathrm{kpc}$, remains unchanged even if we remove the $H_{160}$ magnitude limit on the ALESS sample used for the size measurements. It is also worth emphasizing that this lower bound on the size distribution of SMGs is a conservative limit, as presented in Section 4.2, and the true lower bound on the sizes is likely to be higher, suggesting that more than $60 \%$ of $z=2-3$ SMGs need to go through a significant transitional phase of the stellar distribution.

For any mechanism that drives the transformation of the stellar distribution, it must increase the central stellar masses by either rearranging the stellar mass distribution, forming a significant amount of young stars through bursts of centrally concentrated star formation, or both. A significant intrinsic offset between the dusty star-forming regions and the stellar components of ALESS SMGs, as shown in Section 4.3, means that if ALESS SMGs are isolated, secularly evolving disks, the bulk of the newly formed young stars from the current epoch of star formation need to migrate toward the central regions, which is unlikely given the collisionless nature of the stellar components. On the other hand, recent theoretical work by Dekel \& Burkert (2014) suggests that at $z \sim 2$ rich inflows of pristine gas from the intergalactic medium trigger violent disk instability and dissipatively drive gas into the center, shrinking the gaseous disk into a compact stellar distribution through ongoing star formation, and further evolving into compact quiescent galaxies with high Sérsic indices through various quenching mechanisms. This idea is similar to the bulge formation through migration of long-lived giant clumps in gas- rich disks (e.g., Elmegreen et al. 2008; Perez et al. 2013; Bournaud et al. 2014). However, in order for the inflow of gas to shrink through dissipative processes, the star formation timescale needs to be longer than the inflow timescale, which Dekel \& Burkert (2014) predict to be $250 \mathrm{Myr}$, to avoid turning the majority of the gas into stars before it reaches the center. Unfortunately, the prediction of a long star formation timescale is inconsistent with recent spectroscopic studies of $z \sim 2$ quiescent galaxies, in which spectral line modeling yields stellar populations that have undergone a fast quenching star formation history (SFH) with an $e$-folding timescale of $\tau<250 \mathrm{Myr}$, in the typical exponentially declining SFHs (Kriek et al. 2009; van de Sande et al. 2013; Krogager et al. 2013). Furthermore, for $z \sim 2$ quiescent galaxies, rapidly quenching SFHs not only weaken the likelihood that their progenitors are gaseous disk galaxies and/ or compact star-forming galaxies (Barro et al. 2013; Williams et al. 2014), but also strengthen their connections to $z \sim 2$ SMGs. A similar link between quiescent galaxies and SMGs has also been suggested at $z>3$ based on the short ( $<100 \mathrm{Myr})$ star formation timescale (Marsan et al. 2014). In summary, given the estimated short lifetime of SMGs $(\sim 100 \mathrm{Myr})$, it appears unlikely that the majority of the ALESS SMGs at $z=2-3$ can be significantly transformed into quiescent galaxies with a de Vaucouleurs stellar profile through disk instability.

On the other hand, major galaxy mergers have been shown to efficiently transform stellar distributions from disk-like to de Vaucouleurs's profile through tidal forces and transfer of angular momentum (e.g., Barnes 1988; Barnes \& Hernquist 1996). Recent hydrodynamical simulations have shown that this is also the case for high-redshift gas-rich mergers (Hopkins et al. 2013). Based on Equation (6), by fixing the total $H_{160}$-band fluxes and adopting a typical central surface brightness $\left(\Sigma_{o}\right)$ of $z \sim 2$ quiescent galaxies (median $\Sigma_{o} \sim 17.8$ mag $\operatorname{arcsec}^{-2}$; Szomoru et al. 2012), a simple transformation of Sérsic indices from $n=1$ to $n=4$ would make the effective radius of a source decrease from $4.4 \mathrm{kpc}$ to $\sim 1 \mathrm{kpc}$. If the current burst of star formation doubles the total $H_{160}$-band flux, then the $r_{e}$ would be $\sim 1.4 \mathrm{kpc}$, consistent with the size of $z \sim 2$ quiescent galaxies. Indeed, together with recent IFU dynamical studies, our findings 

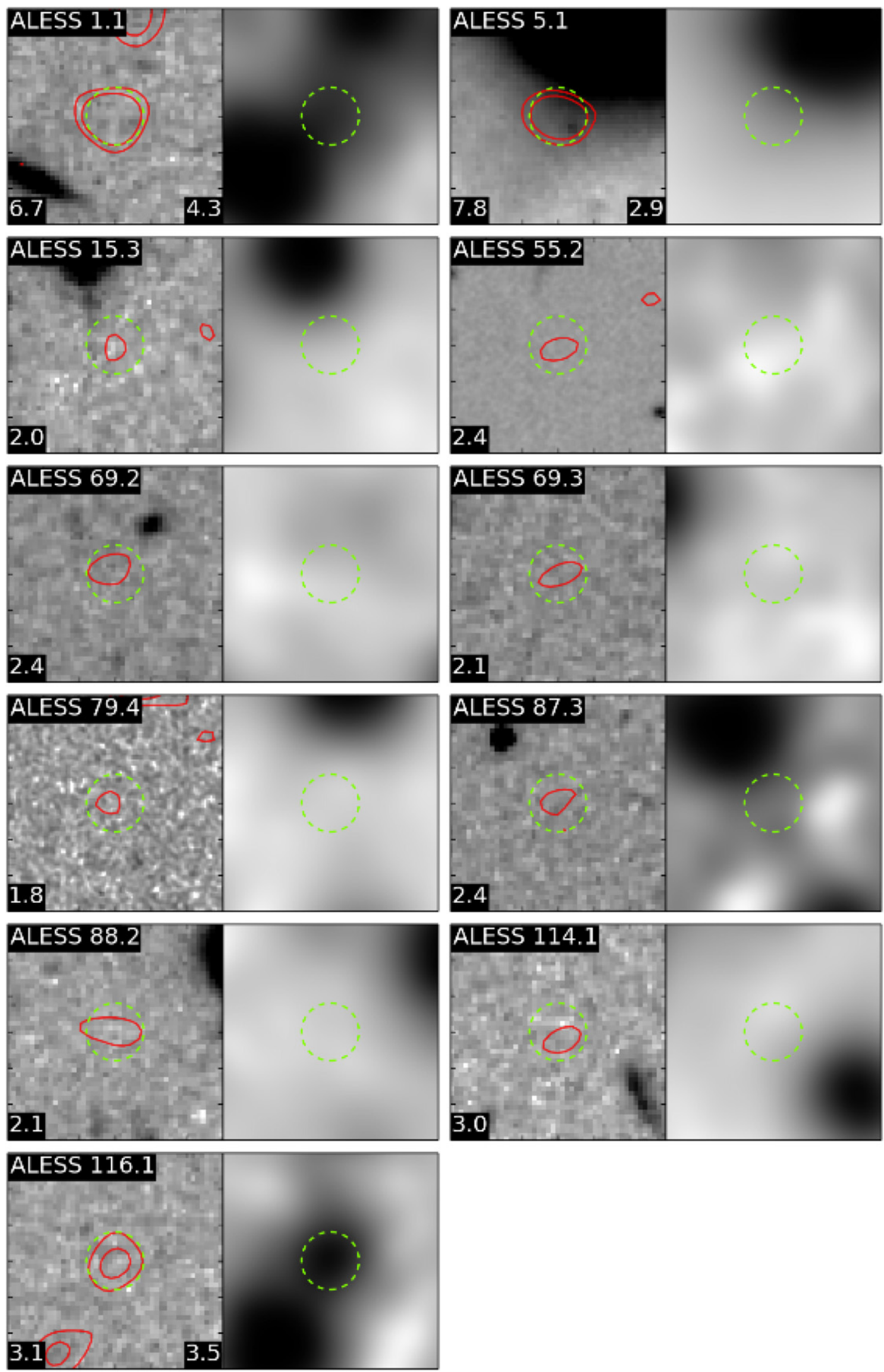

Figure 11. Postage stamps for nondetections of the MAIN ALESS SMGs except ALESS 5.1, which is detected but heavily contaminated by a nearby galaxy, to conduct a Sérsic model fit. Same as Figure 10, but without the middle panels from GALFIT. 

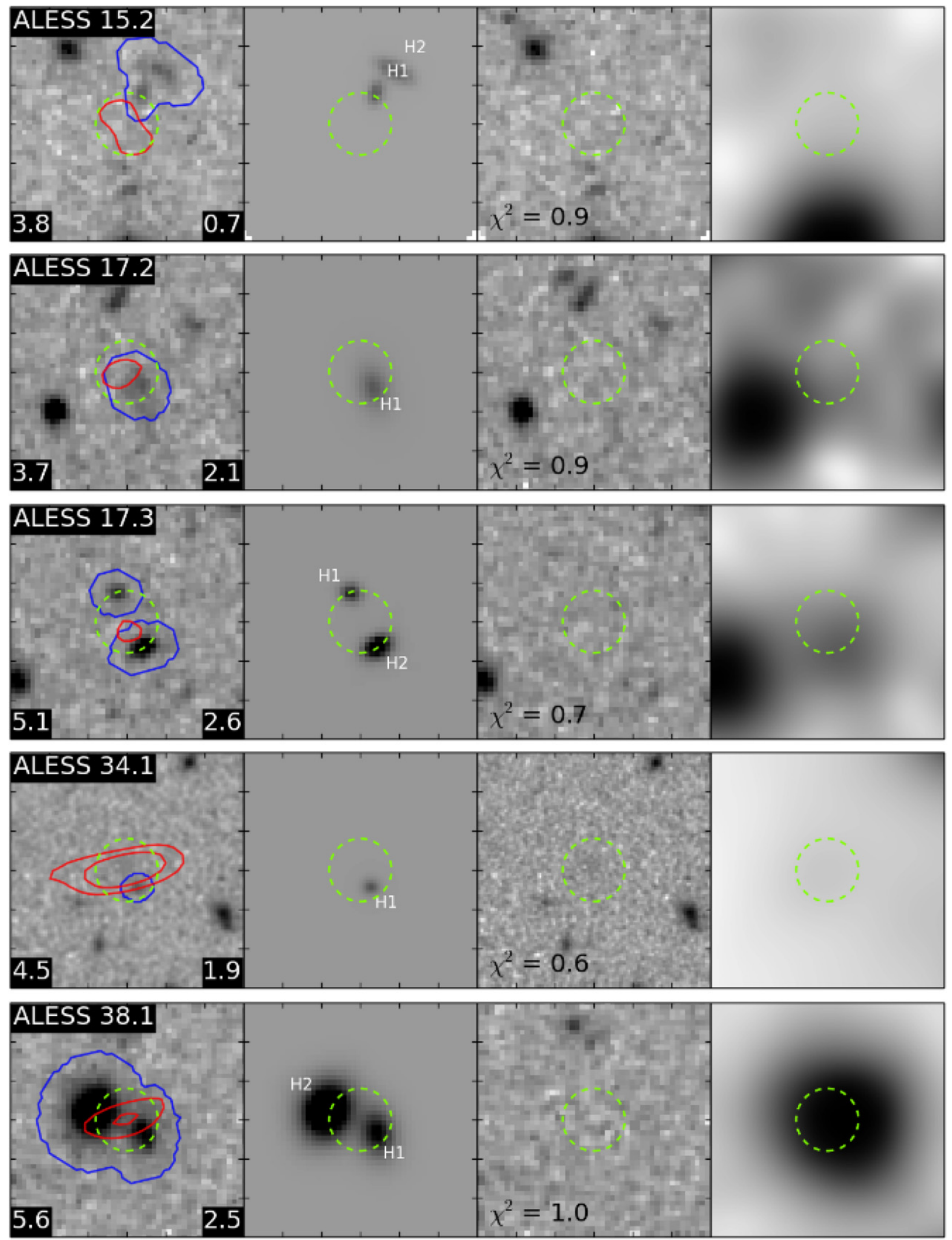

Figure 12. Same as Figure 10, but for the SUPPLEMENTARY ALESS SMGs.

of a high fraction of systems with disturbed stellar morphologies $(\sim 80 \%)$ and significant offsets between star-forming regions and the stellar components suggest specifically that the majority of $z=2-3$ SMGs are likely to be early/mid-stage mergers.

\section{SUMMARY}

We have analyzed $H_{160}$-band imaging taken with the WFC3 camera mounted on the $H S T$ of a sample of 48 ALMA-identified 


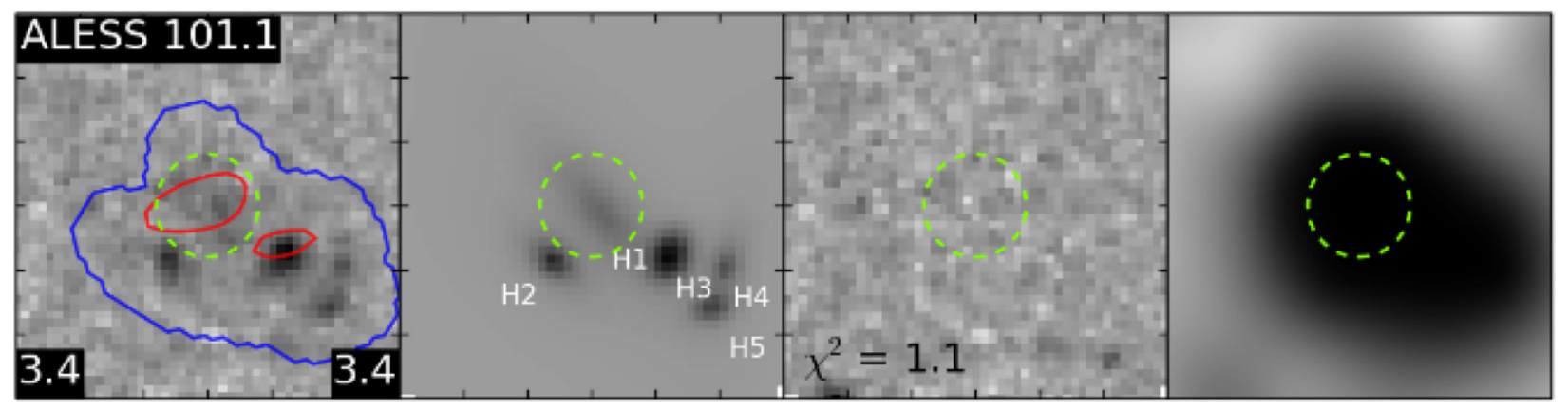

Figure 12. (Continued)
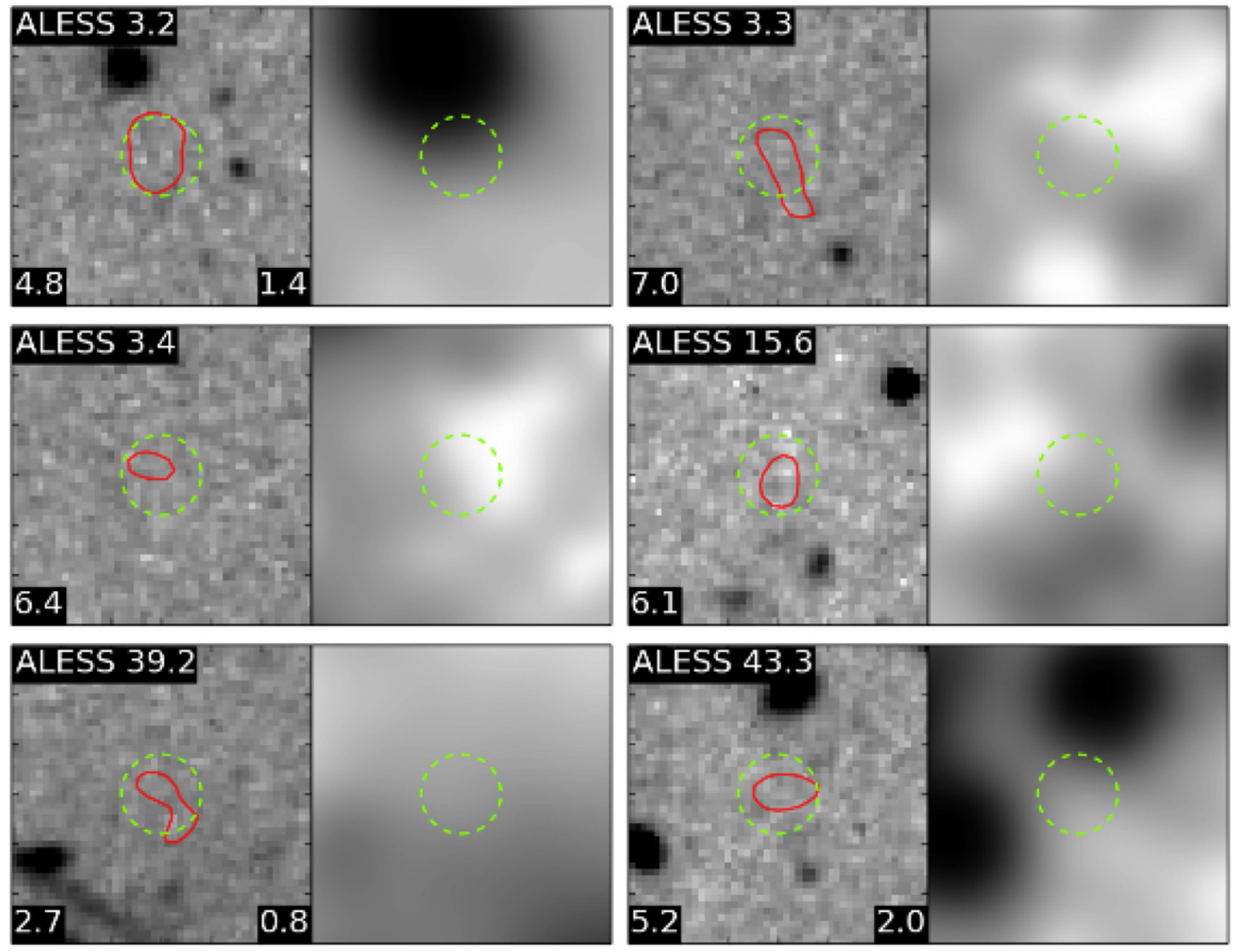

Figure 13. Same as Figure 11, but for the SUPPLEMENTARY ALESS SMGs.

SMGs from the ALESS survey, as well as a comparison sample of 58 ALMA-undetected normal field galaxies at $z=1-3$ located within the ALMA primary beam. (images of all $60 \mathrm{H}_{160^{-}}$ band observed ALESS SMGs can be found in Figures 10-13.) The sample of SMGs is drawn from an interferometric followup study of a flux-limited SMG sample uncovered by the single-dish LABOCA survey at $870 \mu \mathrm{m}$ in ECDFS (LESS survey; Weiß et al. 2009). The key results from our study are as follows.

1. We found that 38 out of 48 ALESS SMGs are detected in the $H_{160}$-band imaging above a typical limiting magnitude of $27.8 \mathrm{mag}$, yielding a detection rate of $79 \% \pm 17 \%$. Most $(80 \%)$ of the nondetections are sources with $S_{870}<3 \mathrm{mJy}$, and about half of the $S_{870}<3$ mJy SMGs are undetected in our $H_{160}$-band imaging. In addition, $61 \% \pm 16 \%$ of the $38 \mathrm{H}_{160}$-detected ALESS SMGs have more than one
$H_{160}$-band component based on our Sérsic profile fitting using GALFIT. In contrast, only $10 \%$ of the comparison sample of lower-luminosity field galaxies has multiple $H_{160}$-band components.

2. We visually classified the $H_{160}$-band morphologies of these galaxies and found that $82 \% \pm 9 \%$ of the $z=1-3$ ALESS SMGs appear to have disturbed morphologies, meaning they are either irregular or interacting systems. In comparison, $48 \% \pm 6 \%$ of the lower-luminosity field sample at similar redshifts appears to be disturbed.

3. Using GALFIT to derive Sérsic profile fits on the $H_{160}$-band imaging, the SMGs at $z=1-3$ have a median Sérsic index of $n=1.2 \pm 0.3$ and a median effective radius (half-light radius) of $r_{e}=4.4_{-0.5}^{+1.1}$. We did not find any correlation among the Sérsic index, effective radius, $L_{\mathrm{IR}}$, redshift, and $H_{160}$. 
4. Since SMGs are likely to be short-lived with an expected lifetime of $\sim 100 \mathrm{Myr}$, along with evidence of fast-quenching SFHs $(\tau<250 \mathrm{Myr})$ for $z \sim 2$ quiescent galaxies, SMGs at $z=2-3$ have many of the properties expected for the progenitors of the $z \sim 2$ quiescent galaxies. Given the claimed fast evolution timescale, we argue that major mergers are the main mechanism that drives the transformation of the stellar distribution, in both effective radius (from $r_{e} \sim 4 \mathrm{kpc}$ to $r_{e}=1-2 \mathrm{kpc}$ ) and Sérsic index (from $n \sim 1$ to $n \sim 4$ ), between $z=2-3$ SMGs and $z \sim 2$ quiescent galaxies. Specifically, our findings that a high fraction of SMGs have a disturbed morphology and significant offsets between the dusty star-forming regions and the stellar distributions suggest that the majority of SMGs at $z=2-3$ are early $/$ mid-stage mergers.

We thank the referee for a very thorough report that improved the manuscript. We thank Jacob Head for useful discussions on GALFIT and Anton Koekemoer for helpful suggestions on the usage of CANDELS maps. This research made use of Astropy, a community-developed core Python package for Astronomy (Astropy Collaboration et al. 2013). This research has made use of NASA's Astrophysics Data System. C.-C.C. and I.R.S. acknowledge support from the ERC Advanced Investigator program DUSTYGAL 321334. I.R.S. also acknowledges support from a Royal Society/Wolfson Merit Award and STFC through grant number ST/L00075X/1. Support for program \#12866 was provided by NASA through a grant from the Space Telescope Science Institute, which is operated by the Association of Universities for Research in Astronomy, Inc., under NASA contract NAS 5-26555. This paper makes use of the following ALMA data: ADS/JAO.ALMA\#2011.1.00294.S. ALMA is a partnership of ESO (representing its member states), NSF (USA), and NINS (Japan), together with NRC (Canada) and NSC and ASIAA (Taiwan), in cooperation with the Republic of Chile. The Joint ALMA Observatory is operated by ESO, AUI/NRAO, and NAOJ.

\section{REFERENCES}

Abraham, R. G., van den Bergh, S., \& Nair, P. 2003, ApJ, 588, 218

Aguirre, P., Baker, A. J., Menanteau, F., Lutz, D., \& Tacconi, L. J. 2013, ApJ, 768,164

Alaghband-Zadeh, S., Chapman, S. C., Swinbank, A. M., et al. 2012, MNRAS, 424, 2232

Alexander, D. M., Bauer, F. E., Chapman, S. C., et al. 2005, ApJ, 632, 736

Aravena, M., Younger, J. D., Fazio, G. G., et al. 2010, ApJL, 719, L15

Astropy Collaboration, Robitaille, T. P., Tollerud, E. J., et al. 2013, A\&A, 558, A33

Barger, A. J., Cowie, L. L., Chen, C.-C., et al. 2014, ApJ, 784, 9

Barger, A. J., Cowie, L. L., Sanders, D. B., et al. 1998, Natur, 394, 248

Barger, A. J., Wang, W.-H., Cowie, L. L., et al. 2012, ApJ, 761, 89

Barnes, J. E. 1988, ApJ, 331, 669

Barnes, J. E., \& Hernquist, L. 1996, ApJ, 471, 115

Barro, G., Faber, S. M., Pérez-González, P. G., et al. 2013, ApJ, 765, 104

Baugh, C. M., Lacey, C. G., Frenk, C. S., et al. 2005, MNRAS, 356, 1191

Bertin, E., \& Arnouts, S. 1996, A\&AS, 117, 393

Biggs, A. D., Ivison, R. J., Ibar, E., et al. 2011, MNRAS, 413, 2314

Bluck, A. F. L., Conselice, C. J., Buitrago, F., et al. 2012, ApJ, 747, 34

Bolzonella, M., Miralles, J.-M., \& Pelló, R. 2000, A\&A, 363, 476

Bothwell, M. S., Smail, I., Chapman, S. C., et al. 2013, MNRAS, 429, 3047

Bournaud, F., Perret, V., Renaud, F., et al. 2014, ApJ, 780, 57

Bouwens, R. J., Illingworth, G. D., Oesch, P. A., et al. 2011, ApJ, 737, 90

Brammer, G. B., van Dokkum, P. G., \& Coppi, P. 2008, ApJ, 686, 1503

Cardamone, C. N., van Dokkum, P. G., Urry, C. M., et al. 2010, ApJS, 189, 270

Casey, C. M., Chen, C.-C., Cowie, L. L., et al. 2013, MNRAS, 436, 1919

Casey, C. M., Narayanan, D., \& Cooray, A. 2014, PhR, 541, 45

Chapman, S. C., Blain, A. W., Smail, I., \& Ivison, R. J. 2005, ApJ, 622, 772
Chapman, S. C., Windhorst, R., Odewahn, S., Yan, H., \& Conselice, C. 2003, ApJ, 599, 92

Charmandaris, V., Laurent, O., Le Floc'h, E., et al. 2002, A\&A, 391, 429

Chen, C.-C., Cowie, L. L., Barger, A. J., et al. 2013, ApJ, 762, 81

Chen, C.-C., Cowie, L. L., Barger, A. J., Wang, W.-H., \& Williams, J. P. 2014, ApJ, 789, 12

Chen, C.-C., Cowie, L. L., Wang, W.-H., Barger, A. J., \& Williams, J. P. 2011, ApJ, 733, 64

Cimatti, A., Cassata, P., Pozzetti, L., et al. 2008, A\&A, 482, 21

Clements, D., Eales, S., Wojciechowski, K., et al. 2004, MNRAS, 351, 447

Conselice, C. J. 2003, ApJS, 147, 1

Conselice, C. J. 2014, ARA\&A, 52, 291

Conselice, C. J., Bluck, A. F. L., Buitrago, F., et al. 2011, MNRAS, 413, 80 Conselice, C. J., Chapman, S. C., \& Windhorst, R. A. 2003, ApJL, 596, L5 Coppin, K. E. K., Swinbank, A. M., Neri, R., et al. 2008, MNRAS, 389, 45 Cowie, L. L., Barger, A. J., Wang, W., \& Williams, J. P. 2009, ApJL, 697, L122 Cowley, W. I., Lacey, C. G., Baugh, C. M., \& Cole, S. 2014, MNRAS, 446, 1784

Damen, M., Labbé, I., van Dokkum, P. G., et al. 2011, ApJ, 727, 1

Danielson, A. L. R., Swinbank, A. M., Smail, I., et al. 2011, MNRAS, 410,1687

Dannerbauer, H., Lehnert, M. D., Lutz, D., et al. 2002, ApJ, 573, 473

Dannerbauer, H., Lehnert, M. D., Lutz, D., et al. 2004, ApJ, 606, 664

Dannerbauer, H., Walter, F., \& Morrison, G. 2008, ApJL, 673, L127

Davé, R., Finlator, K., Oppenheimer, B. D., et al. 2010, MNRAS, 404, 1355

De Breuck, C., Williams, R. J., Swinbank, M., et al. 2014, A\&A, 565, A59

Dekel, A., \& Burkert, A. 2014, MNRAS, 438, 1870

de Vaucouleurs, G. 1948, AnAp, 11, 247

Díaz-Santos, T., Charmandaris, V., Armus, L., et al. 2010, ApJ, 723, 993

Dressel, L. 2014, Wide Field Camera 3 Instrument Handbook, Version 6.0 (available online at http://www.stsci.edu/hst/wfc3/documents/handbooks/ currentIHB/wfc3_cover.html)

Elbaz, D., Dickinson, M., Hwang, H. S., et al. 2011, A\&A, 533, A119

Elmegreen, B. G., Bournaud, F., \& Elmegreen, D. M. 2008, ApJ, 688, 67

Engel, H., Tacconi, L. J., Davies, R. I., et al. 2010, ApJ, 724, 233

Frayer, D. T., Ivison, R. J., Scoville, N. Z., et al. 1998, ApJL, 506, L7

Frayer, D. T., Smail, I., Ivison, R. J., \& Scoville, N. Z. 2000, AJ, 120, 1668

Fu, H., Cooray, A., Feruglio, C., et al. 2013, Natur, 498, 338

Gear, W. K., Lilly, S. J., Stevens, J. A., et al. 2000, MNRAS, 316, L51

Genzel, R., Newman, S., Jones, T., et al. 2011, ApJ, 733, 101

Giavalisco, M. 2002, ARA\&A, 40, 579

Gonçalves, T. S., Basu-Zych, A., Overzier, R., et al. 2010, ApJ, 724, 1373

Graham, A. W., Driver, S. P., Petrosian, V., et al. 2005, AJ, 130, 1535

Greve, T. R., Bertoldi, F., Smail, I., et al. 2005, MNRAS, 359, 1165

Grogin, N. A., Kocevski, D. D., Faber, S. M., et al. 2011, ApJS, 197, 35

Harrison, C. M., Alexander, D. M., Swinbank, A. M., et al. 2012, MNRAS, 426, 1073

Hatsukade, B., Iono, D., Akiyama, T., et al. 2010, ApJ, 711, 974

Hayward, C. C., Behroozi, P. S., Somerville, R. S., et al. 2013, MNRAS, 434

Hayward, C. C., Kereš, D., Jonsson, P., et al. 2011, ApJ, 743, 159

Hickox, R. C., Wardlow, J. L., Smail, I., et al. 2012, MNRAS, 421, 284

Hodge, J. A., Carilli, C. L., Walter, F., et al. 2012, ApJ, 760, 11

Hodge, J. A., Karim, A., Smail, I., et al. 2013, ApJ, 768, 91

Hopkins, P. F., Cox, T. J., Hernquist, L., et al. 2013, MNRAS, 430, 1901

Hopkins, P. F., Cox, T. J., Younger, J. D., \& Hernquist, L. 2009, ApJ, 691, 1168

Hughes, D. H., Serjeant, S., Dunlop, J., et al. 1998, Natur, 394, 241

Iono, D., Peck, A. B., Pope, A., et al. 2006, ApJL, 640, L1

Ivison, R. J., Greve, T. R., Smail, I., et al. 2002, MNRAS, 337, 1

Ivison, R. J., Papadopoulos, P. P., Smail, I., et al. 2011, MNRAS, 412, 1913

Ivison, R. J., Smail, I., Le Borgne, J.-F., et al. 1998, MNRAS, 298, 583

Ivison, R. J., Smail, I., Papadopoulos, P. P., et al. 2010, MNRAS, 404, 198

Karim, A., Swinbank, A. M., Hodge, J. A., et al. 2013, MNRAS, 432, 2

Kartaltepe, J. S., Dickinson, M., Alexander, D. M., et al. 2012, ApJ, 757, 23

Kartaltepe, J. S., Mozena, M., Kocevski, D., et al. 2014, arXiv:1401.2455

Kocevski, D. D., Faber, S. M., Mozena, M., et al. 2012, ApJ, 744, 148

Koekemoer, A. M., Faber, S. M., Ferguson, H. C., et al. 2011, ApJS, 197, 36

Kriek, M., van Dokkum, P. G., Labbé, I., et al. 2009, ApJ, 700, 221

Krist, J. E., Hook, R. N., \& Stoehr, F. 2011, Proc. SPIE, 8127, 16

Krogager, J.-K., Zirm, A. W., Toft, S., Man, A., \& Brammer, G. 2014, ApJ, 797, 17

Larson, D., Dunkley, J., Hinshaw, G., et al. 2011, ApJS, 192, 16

Law, D. R., Steidel, C. C., Shapley, A. E., et al. 2012, ApJ, 745, 85

Lilly, S. J., Eales, S. A., Gear, W. K. P., et al. 1999, ApJ, 518, 641

Lorentz, M. O. 1905, Am. Stat. Assoc., 9, 209

Lotz, J. M., Davis, M., Faber, S. M., et al. 2008, ApJ, 672, 177

Lotz, J. M., Primack, J., \& Madau, P. 2004, AJ, 128, 163

Lutz, D., Dunlop, J. S., Almaini, O., et al. 2001, A\&A, 378, 70 
Marsan, Z. C., Marchesini, D., Brammer, G. B., et al. 2014, arXiv:1406.0002 Menéndez-Delmestre, K., Blain, A. W., Smail, I., et al. 2009, ApJ, 699, 667 Menéndez-Delmestre, K., Blain, A. W., Swinbank, M., et al. 2013, ApJ, 767,151

Morishita, T., Ichikawa, T., \& Kajisawa, M. 2014, ApJ, 785, 18

Mortlock, A., Conselice, C. J., Hartley, W. G., et al. 2013, MNRAS, 433, 1185

Newman, A. B., Ellis, R. S., Bundy, K., \& Treu, T. 2012, ApJ, 746, 162

Oke, J. B., \& Gunn, J. E. 1983, ApJ, 266, 713

Patel, S. G., van Dokkum, P. G., Franx, M., et al. 2013, ApJ, 766, 15

Peng, C. Y., Ho, L. C., Impey, C. D., \& Rix, H.-W. 2010, AJ, 139, 2097

Perez, J., Valenzuela, O., Tissera, P. B., \& Michel-Dansac, L. 2013, MNRAS, 436, 259

Poglitsch, A., Waelkens, C., Geis, N., et al. 2010, A\&A, 518, L2

Pope, A., Borys, C., Scott, D., et al. 2005, MNRAS, 358, 149

Riechers, D. A., Hodge, J., Walter, F., Carilli, C. L., \& Bertoldi, F. 2011, ApJL, 739, L31

Robertson, B., Bullock, J. S., Cox, T. J., et al. 2006, ApJ, 645, 986

Robertson, B. E., \& Bullock, J. S. 2008, ApJL, 685, L27

Rujopakarn, W., Rieke, G. H., Eisenstein, D. J., \& Juneau, S. 2011, ApJ, 726, 93

Sakamoto, K., Ho, P. T. P., \& Peck, A. B. 2006, ApJ, 644, 862

Sakamoto, K., Wang, J., Wiedner, M. C., et al. 2008, ApJ, 684, 957

Sersic, J. L. 1968, Atlas de galaxias australes (Cordoba: Observatorio Astronomico de Cordoba)

Simpson, J. M., Smail, I., Swinbank, A. M., et al. 2012, MNRAS, 426, 3201

Simpson, J. M., Swinbank, A. M., Smail, I., et al. 2014, ApJ, 788, 125

Smail, I., Chapman, S. C., Blain, A. W., \& Ivison, R. J. 2004, ApJ, 616, 71

Smail, I., Ivison, R. J., \& Blain, A. W. 1997, ApJL, 490, L5

Smail, I., Ivison, R. J., Blain, A. W., \& Kneib, J.-P. 1998, ApJL, 507, L21

Smail, I., Ivison, R. J., Owen, F. N., Blain, A. W., \& Kneib, J. 2000, ApJ, 528,612
Smolčić, V., Aravena, M., Navarrete, F., et al. 2012a, A\&A, 548, 4 Smolčić, V., Navarrete, F., Aravena, M., et al. 2012b, ApJS, 200, 10 Swinbank, A. M., Chapman, S. C., Smail, I., et al. 2006, MNRAS, 371, 465 Swinbank, A. M., Lacey, C. G., Smail, I., et al. 2008, MNRAS, 391, 420 Swinbank, A. M., Papadopoulos, P. P., Cox, P., et al. 2011, ApJ, 742, 11 Swinbank, A. M., Simpson, J., Smail, I., et al. 2014, MNRAS, 438, 1267 Swinbank, A. M., Smail, I., Chapman, S. C., et al. 2010, MNRAS, 405, 234 Szomoru, D., Franx, M., \& van Dokkum, P. G. 2012, ApJ, 749, 121 Tacconi, L. J., Genzel, R., Smail, I., et al. 2008, ApJ, 680, 246 Tacconi, L. J., Neri, R., Chapman, S. C., et al. 2006, ApJ, 640, 228 Targett, T. A., Dunlop, J. S., Cirasuolo, M., et al. 2013, MNRAS, 432, 2012 Toft, S., Smolčić, V., Magnelli, B., et al. 2014, ApJ, 782, 68

Trujillo, I., Conselice, C. J., Bundy, K., et al. 2007, MNRAS, 382, 109 Ueda, J., Iono, D., Yun, M. S., et al. 2014, ApJS, 214, 1 van der Wel, A., Franx, M., van Dokkum, P. G., et al. 2014, ApJ, 788, 28 van de Sande, J., Kriek, M., Franx, M., et al. 2013, ApJ, 771, 85 van Dokkum, P. G., Franx, M., Kriek, M., et al. 2008, ApJL, 677, L5 van Dokkum, P. G., Whitaker, K. E., Brammer, G., et al. 2010, ApJ, 709, 1018 Wang, S. X., Brandt, W. N., Luo, B., et al. 2013, ApJ, 778, 179

Wang, W., Cowie, L. L., Barger, A. J., \& Williams, J. P. 2011, ApJL, 726, L18 Wang, W., Cowie, L. L., van Saders, J., Barger, A. J., \& Williams, J. P. 2007, ApJL, 670, L89

Wardlow, J. L., Smail, I., Coppin, K. E. K., et al. 2011, MNRAS, 415, 1479

Weiß, A., Kovács, A., Coppin, K., et al. 2009, ApJ, 707, 1201

Whitaker, K. E., Kriek, M., van Dokkum, P. G., et al. 2012, ApJ, 745, 179

Wiklind, T., Conselice, C. J., Dahlen, T., et al. 2014, ApJ, 785, 111

Williams, C. C., Giavalisco, M., Cassata, P., Tundo, E., et al. 2014, ApJ, 780, 1 Younger, J. D., Fazio, G. G., Huang, J., et al. 2007, ApJ, 671, 1531

Younger, J. D., Fazio, G. G., Huang, J.-S., et al. 2009, ApJ, 704, 803

Younger, J. D., Fazio, G. G., Wilner, D. J., et al. 2008, ApJ, 688, 59

Yun, M. S., Scott, K. S., Guo, Y., et al. 2012, MNRAS, 420, 957 